\author{
UNIVERSIDADE DE SÃO PAULO \\ FACULDADE DE ODONTOLOGIA DE BAURU
}

CRISTIANE MARQUES DORCE

Avaliação de dispositivo para viabilização de Análise de Frequência de Ressonância em Implantes Dentários em Ovelhas 



\title{
Avaliação de dispositivo para viabilização de Análise de Frequência de Ressonância em Implantes Dentários em Ovelhas
}

\author{
Dissertação constituída por artigo apresentada à \\ Faculdade de Odontologia de Bauru da \\ Universidade de São Paulo para obtenção do título \\ de Mestre em Ciências no Programa de Ciências \\ Odontológicas Aplicadas, na área de concentração \\ Reabilitação Oral. \\ Orientador: Prof. Dr. Accácio Lins do Valle \\ Co-orientador: Prof. Dr. Carlos dos Reis Pereira de \\ Araujo
}


Dorce, Cristiane Marques

D738a Avaliação de dispositivo para viabilização de Análise de Frequência de Ressonância em Implantes Dentários em Ovelhas / Cristiane Marques Dorce - Bauru, 2018.

81 p. : il. ; $30 \mathrm{~cm}$.

Dissertação (Mestrado) - Faculdade de Odontologia de Bauru. Universidade de São Paulo

Orientador: Prof. Dr. Accácio Lins do Valle Co-orientador: Prof. Dr. Carlos dos Reis Pereira de Araujo

Autorizo, exclusivamente para fins acadêmicos e científicos, a reprodução total ou parcial desta dissertação por processos fotocopiadores e outros meios eletrônicos.

Assinatura: 
FOLHA DE APROVAÇÃO 



\section{DEDICATÓRIA}

À Deus, pela proteção, por abrir os caminhos em meios às adversidades, por me tornar capaz, por me fazer confiante em momentos em que eu não acreditava, por Sua voz ser mais forte frente aos turbilhões que passei para chegar até aqui.

Ao meu pai, Vanildo Aparecido Dorce, por acreditar em mim, por me apoiar em todos os meus projetos e sonhos, por me ensinar, que nunca devemos desistir daquilo que acreditamos com esforço para ser o melhor que eu puder, naquilo que escolhi ser.

À minha mãe, Eva Cristina Marques Dorce, pelas orações, pelo colo, por enxugar minhas lágrimas, por ser minha companheira, amiga, por não deixar que eu desistisse dos meus sonhos, por me mostrar que seria possivel, por ser um exemplo de força e coragem, por cuidar tão bem da minha família, sendo o nosso alicerce.

À minha irmã, Maria Eduarda Marques Dorce por ser aquela que me motiva a me esforçar, a ser melhor, para ser seu exemplo quando as adversidades em sua vida chegarem. Pelo sorriso, pelo consolo, pelo carinho mesmo quando não entendia porque eu "estudava tanto".

Aos amigos e familiares, todos que me apoiaram de alguma forma, que me consolaram, que não me deixaram desistir, e que acreditaram em mim nesse momento, por serem "anjos" na minha vida.

Amo vocês! 



\section{AGRADECIMENTOS}

Ao Prof. Dr. Accácio Lins do Valle, por me receber como sua orientada, pela sua disponibilidade, pela calma, paciência, por todas ajudas em clínica, pelo cuidado que foi fundamental para tornar o meu caminho até aqui possível. A minha admiração e gratidão!

Ao Prof. Dr. Carlos dos Reis Pereira de Araujo, pela co-orientação, por todos os ensinamentos e experiências adquiridas, por viabilizar experimentos em parceria com Pirassununga, além de recursos para que o estudo fosse possível. A minha gratidão!

Ao Prof. Dr. Pedro César Garcia de Oliveira, pelo carinho, apoio e por toda ajuda na clínica. Além de professor, foi um amigo, me incentivando constantemente nos momentos mais delicados. O meu carinho e gratidão!

Ao Prof. Dr. Luiz Fernando Pegoraro, sou grata pelo conhecimento transmitido durante os seminários e clínicas, à constante exigência durante o mestrado, mas também pela compreensão, pelo carinho comigo. Sendo mais que professor de prótese, foi meu professor de vida, contribuindo para meu crescimento profissional e pessoal. A minha gratidão e admiração!

Ao Prof. Dr. Estevam Augusto Bonfante por ter me incentivado desde a minha graduação, pelo companheirismo, pelos conhecimentos transmitidos, pelo seu exemplo de vida e por seu incrível dom de empatia com os alunos. A minha gratidão e admiração!

A todos os demais professores do Departamento de Prótese da FOB-USP: Ana Lúcia Pompéia Fraga de Almeida, Karin Hermana Neppelenbroek, Lucimar Falavinha Vieira, José Henrique Rubo, Paulo Conti, Vinícius Carvalho Porto, Renato de Freitas. Agradeço por compartilharem seus conhecimentos desde a Graduação e também durante os seminários e clínicas. Não há palavras para descrever todo meu aprendizado durante todo o convívio no departamento.

Aos meus colegas de pós-graduação: Ana Paula Chappuis, Cindy Ruiz, Dyna Mara Ferreira, Ernesto Benalcázar, Camila Perfeito pelo companheirismo, dificuldades e alegrias divididas. 

Às minhas amigas Camila Machado, Leadiane Sandes e Jéssica Moreno, que se tornaram minha base durante os anos de Mestrado, pelo apoio, incentivo, pela amizade que levarei para sempre comigo! Muito obrigada!

Aos funcionários, pela receptividade e convivência harmoniosa, em especial a Cleide, Débora, Hebe e Cleuza que sempre estiveram disponíveis para ajudar, pelo acolhimento e pelo carinho.

À Faculdade de Odontologia de Bauru, Universidade de São Paulo. É uma honra ser aluna de graduação e pós-graduação desta faculdade, que permitiu meu crescimento como pessoa e como profissional. Todas as experiências adquiridas nesse lugar me mostram que estudar vale a pena, que a Educação transforma realidades e que valeu a pena todo o esforço para pertencer a esse lugar. Quanto orgulho!

Ao Conselho Nacional de Desenvolvimento Científico e Tecnológico (CNPq) pela bolsa de Mestrado, que tornou possível a realização desse mestrado.

A todos que contribuíram de alguma forma, Muito obrigada! 

"Conheça todas as teorias, domine todas as técnicas, mas ao tocar uma alma humana, seja apenas outra alma humana"

Carl Jung 




\section{RESUMO}

\section{Avaliação de dispositivo para viabilização de análise de frequência de ressonância em Implantes Dentários em Ovelhas}

Afirmação do problema. Estudos experimentais em animais são utilizados para avaliar previamente o resultado de pesquisas e sua necessidade de serem realizadas em humanos. Estudos em animais são fundamentais na implantodontia para que haja uma melhor compreensão da osseointegração. Durante o processo de execução de pesquisas nesse tipo de estudo, algumas dificuldades relacionadas ao manuseio, custos de manutenção e bem-estar dos animais tendem a dificultar o seu desenvolvimento.

Finalidade. Esse estudo tem como objetivo, testar um novo dispositivo acoplado sobre o implante, para que possa atuar como uma extensão do mesmo, facilitando a realização da Análise de Frequência de Ressonância durante o processo de osseointegração permitindo que implantes instalados em ovelhas em diferentes períodos, possam ser reabertos sem que haja a necessidade de etapas cirúrgicas para a reabertura durante a realização de testes.

Material e métodos. Foram utilizadas 4 ovelhas, sendo uma do Grupo Controle (GC) e 3 do Grupo Teste (GT). Cada animal recebeu oito implantes localizados próximos ao ângulo da mandíbula, sendo quatro do lado esquerdo e quatro do lado direito, totalizando, 32 implantes cone-morse Acqua ${ }^{\circledR}$ de 3,5 de diâmetro por 8,5mm de comprimento da empresa Neodent ${ }^{\circledR}$ que foram avaliados pela análise de frequência de ressonância com a medição do ISQ (Quociente de Estabilidade do Implante) utilizando um transdutor (SmartPeg) expressos numa escala de 0 a 100. Para o Grupo Controle (GC) as medições de ISQ foram realizadas pelo método convencional, onde o SmartPeg foi acoplado diretamente sobre a plataforma do implante. Já no Grupo Teste (GT) o dispositivo estudado, foi acoplado ao implante para que pudesse ser realizada a medição. Os períodos estudados foram: imediatamente após a cirurgia e com quatro semanas de osseointegração. Para a análise estatística foram empregados os testes de Wilcoxon e Man-Whitney com a probabilidade de $5 \%(p<0.05)$. 

Resultados. As médias obtidas no GC foram 50,47 imediatamente após cirurgia e 65,68 após 4 semana, enquanto que no GT foram 34,74 e 34,81, respectivamente. Comparando a diferença entre os grupos nas médias de ISQ imediatas e após quatro semanas, houve diferença significativa com $p=0.0321$ e $p=0.0027$, respectivamente, demonstrando que os resultados no GT nos dois períodos não foram equivalentes quando comparados ao GC.

Conclusão. Os resultados demonstraram que o grupo teste, apesar de trazer benefícios em relação ao manuseio dos animais, não apresentou benefícios com relação à eficiência na leitura do ISQ, quando comparado ao método convencional.

Palavras chave: Implante dentário. Osseointegração. Quociente de estabilidade do implante 

ABSTRACT 



\begin{abstract}
Desenvolvimento de dispositivo para viabilização de testes de torque e destorque e análise de frequência de ressonância em Implantes Dentários em Modelo animal "Ovelha"
\end{abstract}

Statement of the problem. Experimental studies in animals are used to pre-evaluate the results of research and their need to be performed in humans. Animal studies are fundamental in implantology so that there is a better understanding of osseointegration. During the execution of research in this type of study, some difficulties related to handling, maintenance costs and animal welfare tend to hinder its development.

Purpose. This study aims to test a new device coupled on the implant, so that it can act as an extension of the same, facilitating the performance of the Resonance Frequency Analysis during the osseointegration process allowing implants installed in different periods of time, can be reopened without the need for surgical steps for reopening during testing.

Material and methods. Four sheep were used, one being from the Control Group (GC) and 3 from the Test Group (GT). Each animal received eight implants located near the angle of the mandible, four on the left side and four on the right side, totaling 32 Acqua $\AA 3.5 \mathrm{~mm}$ diameter $8.5 \mathrm{~mm}$ long implants of the company Neodent $\AA$ that were evaluated by the resonance frequency analysis with the ISQ (Implant Stability Ratio) measurement using a transducer (SmartPeg) expressed on a scale of 0 to 100. For the Control Group (GC) the ISQ measurements were performed by the conventional method, where the SmartPeg was attached directly onto the implant platform. In the Test Group (GT) the studied device was attached to the implant so that the measurement could be performed. The periods studied were: immediately after surgery and four weeks of osseointegration. For the statistical analysis Wilcoxon and Man-Whitney tests were used, with a probability of $5 \%(p<0.05)$.

Results. The averages obtained in the CG were 50.47 immediately after surgery and 65.68 after 4 weeks, while in the GT were 34.74 and 34.81 , respectively. Comparing the difference between the groups in the means of immediate SAI and after four 

weeks, there was a significant difference with $p=0.0321$ and $p=0.0027$, respectively, demonstrating that the results in the WG in the two periods were not equivalent when compared to the $\mathrm{CG}$.

Conclusion. The results showed that the test group, despite having benefits in relation to the handling of the animals, did not present benefits in relation to the efficiency of the ISQ reading when compared to the conventional method.

Key words: Dental implant. Osseointegration. Implant Stability Quotient. 



\section{LISTA DE ILUSTRAÇÕES}

Figura 1 - Desenho para fabricação do componente para implantes cone morse

Figura 2 - Componente após sua fabricação e antes de ser instalado sobre a plataforma do implante cone morse

Figura 3 - Dispositivo Osstell ISQ ${ }^{\mathrm{TM}}$

Figura 4 - Demonstração do acoplamento do SmartPeg ao Componente

Figura 5 - Instalação do SmartPeg à plataforma do implante e obtenção do ISQ pelo transdutor Osstell ${ }^{\mathrm{TM}}$

Figura 6 - Instalação de cicatrizadores de $3,5 \mathrm{~mm}$ sobre a plataforma dos implantes cone morse.

Figura 7 - Aspecto após sutura na Ovelha Controle

Figura 8 - Medição do ISQ na Ovelha Controle, após reabertura dos implantes, remoção dos cicatrizadores e acoplamento do SmartPeg. 46

Figura 9 - Instalação do componente após instalação dos implantes no Grupo Teste

Figura 10 - Aspecto cirúrgico após sutura, demonstrando a extensão externa dos componentes numa vista aproximada

Figura 11 - Aspecto cirúrgico após sutura, demonstrando a extensão dos componentes numa visão ampliada, demonstrando a região do ângulo da mandíbula da ovelha

Figura 12 - Gráfico em Boxplot geral, demonstrando médias de ISQ dos grupos Teste e Controle imediatamente após instalação dos implantes e após 4 semanas 



\section{LISTA DE TABELAS}

Tabela 1 - Valores médios de ISQ no grupo Controle imediatamente após instalação dos implantes e após 4 semanas

Tabela 2 - Valores médios de ISQ no grupo Teste imediatamente após instalação dos implantes e após 4 semanas 



\section{ÍNDICE}

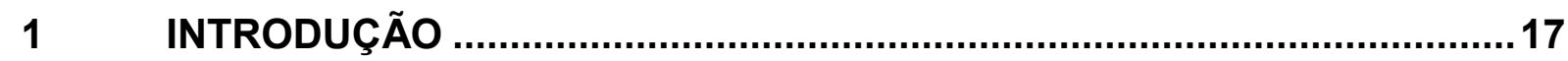

2 ARTIGO

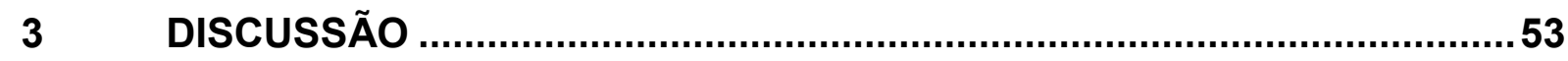

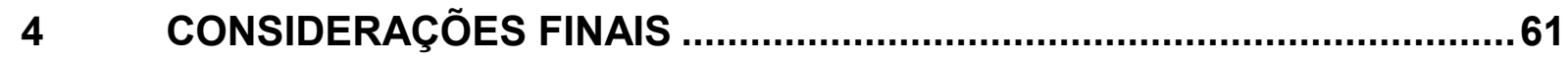

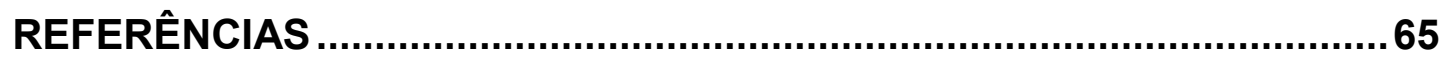

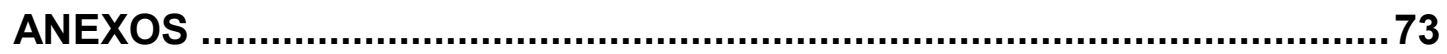



1 INTRODUÇÃO 



\section{INTRODUÇÃO}

Os implantes dentários correspondem a uma grande parcela do mercado de cuidados com a saúde, que cresce rapidamente ${ }^{1}$, tornando-se uma opção comum em tratamentos de Reabilitação Oral em pacientes edêntulos parciais ou totais. ${ }^{2}$

O sucesso de um implante depende do contato direto entre ele e o tecido ósseo e, determinando uma osseointegração, na qual promove uma interface, sem a presença de tecido conjuntivo interposto entre ambos, criando uma ancoragem mecanicamente firme. ${ }^{3}$

Existem dois processos na formação óssea ao redor dos implantes:

-Osteogênese de contato: formação óssea em contato direto com a superfície do implante, que também pode ser denominada "estabilidade primária", que é a fixação inicial do implante ao tecido ósseo durante a cirurgia.

-Osteogênese à distância: ocorre uma nova formação óssea, quando não foi obter uma fixação firme e estável no momento da cirurgia, sendo denominada "estabilidade secundária". O espaço entre a superfície do implante e osso, fica preenchido por um coágulo que gradualmente será substituído por uma matriz orgânica, que atuará como um arcabouço para a formação do tecido ósseo.

A taxa de sobrevivênciade um implante está relacionada a sua estabilidade primária porém, sugere-se que uma adequada osseointegração possa ocorrer mesmo que não havendo a fixação inicial ${ }^{4,5}$. Outros fatores poderão influenciar na osseointegração como: o tipo e o formato do osso remanescente, o posicionamento do implante com dimensões de osso adequadas, tempo de cicatrização óssea, o material utilizado, comprimento, diâmetro e tratamento de superfície do implante ${ }^{6,7}$.

As propriedades superficiais dos implantes estão sendo cada vez mais estudadas por pesquisadores $8,9,10$. Dentre elas podemos citar: composição química, a topografia; energia superficial; rugosidade e a molhabilidade (ou seja, quando um implante entra em contato com um meio aquoso). Tais propriedades podem 
contribuir para a fixação de íons e proteínas à superfície dos implantes, facilitando a interação e desenvolvimento celular e tecidual na interface organismo/biomaterial

Pesquisas atuais buscam por modificações estruturais nos implantes para melhorias da resposta inicial entre hospedeiro/implante e a redução no tempo de tratamento. Os benefícios clínicos seriam: reabilitações protéticas mais rápidas e um melhor desempenho em regiões de baixa qualidade óssea ${ }^{11}$.

Estudos experimentais em animais são necessários para previamente avaliar o resultado de pesquisas e possivelmente verificando a necessidade ou não de serem realizadas em humanos.

É importante a avaliação dos estudos de implante, osso e articulação para compreender os conceitos básicos da estrutura óssea e da vantagens e desvantagens dos diferentes modelos animais. Embora nenhum modelo seja ideal, várias espécies podem fornecer suporte para pesquisas².

As análises "in vitro" dão informações sobre a compatibilidade e toxicidade de um biomaterial assim como o comportamento celular, evitando o uso desnecessário de animais quando o material testado é citologicamente inadequado. Os modelos experimentais em animais têm sido utilizados na pesquisa biomédica, pois os modelos "in vitro" não conseguem reproduzir completamente a complexidade do corpo humano, sendo assim, permitem o desenvolvimento de conhecimentos básicos que apoiem os estudos pré-clinicos e clínicos, tendo como objetivo a coleta de informações relevantes para compreensão da biologia, bem-estar de seres humanos e animais, além de melhorias na manutenção da saúde ${ }^{1}$.

Após a etapa laboratorial, os estudos "in vivo" avaliarão: biocompatibilidade, capacidade regenerativa dos materiais, dispositivos de fixação de enxertos ósseos a curto e médio prazo bem como a osseointegração dos implantes ${ }^{12}$.

A avaliação dos implantes in vivo tem como finalidade avaliar:

-Relevância Biológica: testes iniciais para demonstrar a relevância da estrutura e composição do material. São informações iniciais sobre, como um implante pode afetar as funções fisiológicas, biomecânicas e hormonais do tecido 
ósseo. Esse estágio geralmente utiliza modelos animais de pequeno porte como roedores e coelhos.

-Biofuncionalidade: são testes que identificam a capacidade de um implante para alterar a função do tecido de maneira desejada como a condrogênese, osseointegração, osteocondutividade e osteoindução. Esses testes são utilizados em roedores e coelhos, mas têm uma limitação quanto ao tamanho dos implantes em relação ao animal, por isso, também são realizadas em cães, porcos e pequenos ruminantes.

-Biocompatibilidade e Segurança: testes para identificar efeitos locais ou sistêmicos indesejados. Os implantes não podem ser: tóxicos, trombogênicos, carcinogênicos, antigênico e mutagênicos. Geralmente são utilizados em roedores e coelhos.

-Relevância e Eficácia Clínica: etapa é necessária demonstrar o funcionamento dos implantesem animais maiores como cães, pequenos ruminantes, porcos ou primatas não humanos, onde o fluxo de sangue local e carregamento mecânico podem ser críticos para seu sucesso do implante.

Esses testes em modelos animais, clinicamente relevantes é um aspecto importante da pesquisa e, na maioria dos casos, requisito fundamental do início da experimentação em humanos ${ }^{13}$. Embora nenhum modelo seja ideal, várias espécies podem fornecer suporte para pesquisas. Muitos modelos animais foram empregados para cicatrização intra e extra oral do osso em torno dos implantes dentários, incluindo ovelhas como modelos ${ }^{2}$.

O modelo animal "ovelha" tem crescido nas pesquisas da Implantodontia, sendo utilizadas para modelar defeitos em ossos longos, testar materiais em defeitos cranianos, resposta tecidual e abordagens cirúrgicas ${ }^{1,14}$.

Pesquisas recentes demonstraram a utilização das ovelhas para pesquisas em implantodontia ${ }^{2,15,16}$, sendo que alguns autores utilizam esses implantes no ângulo da mandíbula desses animais, modelo esse que foi utilizado em nossa pesquisa $^{17,18,19}$. 
Os implantes osseointegrados são clinicamente caracterizados pela ausência de mobilidade, que pode ser avaliada pela medição da estabilidade primária e secundária do implante ${ }^{20}$.

Métodos de testes mecânicos como torque inverso ou "destorque", foram utilizados para estudar e medir essa interface mecânica entre implante e osso ${ }^{3}$. Mas alguns métodos e técnicas para medir a estabilidade do implante envolvem análises quantitativas não invasivas, não prejudicando a interface ósseo-implante medindo a estabilidade do implante de forma objetiva, por exemplo, análise de freqüência de ressonância (RFA), como é o utilizado pelo dispositivo Osstell TM.

A Análise de Freqüência de Ressonância foi utilizada para documentar mudanças na cicatrização óssea ao longo da interface implante-osso, medindo a rigidez do implante no tecido ósseo, sendo também utilizado para determinar se os implantes estão prontos para a restauração final ou receberem cargae identificar os implantes em "risco".

Os primeiros estudos com RFA foram publicados em 199621. No ano de 1997, Meredith et al et al. ${ }^{22}$ sugeriu um método não-invasivo para determinar a freqüência de ressonância associada aos implantes dentários, conectando um adaptador/transdutor à plataforma de implantes nos estudos com animais. O sistema RFA baseado em pulsos magnéticos, foi comercialmente produzido como Osstell desde o ano 2000 (Osstell AB, Gotemburgo, Suécia). Recebeu essa denominação devido ao seu idealizador, seguido por Osstell Mentor TM e Osstell ISQ TM. No método RFA, um sensor "smartpeg" é acoplado ao implante, e subsequentemente a ponta do dispositivo é mantida próxima ao sensor durante o período de emissão de impulsos eletromagnéticos. Os valores de freqüência de ressonância são automaticamente medidos em unidades de kilohertz e convertidos em uma escala chamada Quociente de Estabilidade do Implante (ISQ) exibida no dispositivo. Os valores de ISQ variam de 1 a 100 , e quanto maior o ISQ, maior a estabilidade do implante indicando uma maior rigidez óssea. ${ }^{23,24,25}$.

Esse estudo teve como objetivo testar um dispositivo extensor de $6 \mathrm{~mm}$ que é rosqueado ao implante, reproduzindo a conexão cônica com precisão, permitindo o acoplamento e a utilização de um SmartPeg padrão Osstell ISQ ${ }^{\mathrm{TM}}$ permitindo que os 
testes de análise de frequência de ressonância sejam realizados sem intervenções cirúrgicas pós implantação de implantes nos animais preservando a saúde e integridade do animal. 

2 ARTIGO 



\section{ARTIGO}

O artigo presente nesta Dissertação foi escrito de acordo com as instruções e diretrizes para submissão de artigos do Journal of Prosthetic Dentistry (Anexo B). 


\section{AVALIAÇÃO DE DISPOSITIVO PARA VIABILIZAÇÃO DE TESTES DE ANÁLISE DE FREQUÊNCIA DE RESSONÂNCIA EM IMPLANTES DENTÁRIOS EM OVELHAS}

\section{RESUMO}

Afirmação do problema. Na Implantodontia, experimentos em animais têm fundamental importância para uma melhor compreensão do processo de osseointegração. Algumas dificuldades são encontradas durante a realização desse tipo de experimento, relacionado ao manuseio, custos e bem-estar do animal.

Finalidade. Esse estudo tem como objetivo testar um novo dispositivo acoplado sobre a plataforma do implante, servindo como uma extensão do mesmo, permitindo assim a realização testes mecânicos durante o processo de osseointegração de implantes instalados em ovelhas em vários períodos, dispensando etapas cirúrgicas de reabertura do implante para realização dos testes.

Material e métodos. Nesse estudo foram utilizadas 4 ovelhas, sendo uma do Grupo Controle (GC) e 3 do Grupo Teste (GT). Cada animal recebeu oito implantes localizados próximos ao ângulo da mandíbula, sendo quatro do lado esquerdo e quatro do lado direito, totalizando, 32 implantes cone-morse Acqua ${ }^{\circledR}$ de 3,5 de diâmetro por $8,5 \mathrm{~mm}$ de comprimento da empresa Neodent ${ }^{\circledR}$ que foram avaliados pela análise de frequência de ressonância com a medição do ISQ (Quociente de Estabilidade do Implante) utilizando um transdutor (SmartPeg) expressos numa escala de 0 a 100. Para o Grupo Controle (GC) as medições de ISQ foram realizadas pelo método convencional, onde o SmartPeg foi acoplado diretamente sobre a plataforma do implante. Já no Grupo Teste (GT) o dispositivo estudado, foi acoplado ao implante para que pudesse ser realizada a medição. Os períodos estudados foram: imediatamente após a cirurgia e com quatro semanas de osseointegração. Para a análise estatística foram empregados os testes de Wilcoxon e Man-Whitney com a probabilidade de $5 \%(p<0.05)$.

Resultados. As médias obtidas no GC foram 50,47 imediatamente após cirurgia e 65,68 após 4 semana, enquanto que no GT foram 34,74 e 34,81, respectivamente. Comparando a diferença entre os grupos nas médias de ISQ imediatas e após quatro semanas, houve diferença significativa com $p=0.0321$ e $p=0.0027$, 
respectivamente, demonstrando que os resultados no GT nos dois períodos não foram equivalentes quando comparados ao GC.

Conclusão. Os achados demonstram que o grupo teste, apesar de trazer benefícios para a pesquisa, não demonstrou equivalência nos resultados nas medições de ISQ quando comparados ao método convencional.

Novos dispositivos devem ser desenvolvidos com a finalidade de simplificar pesquisas complexas, reduzir os custos, assim como preservar o bem-estar dos animais.

Palavras chave: Implante Dentário. Osseointegração.

\section{INTRODUÇÃO}

Os implantes dentários correspondem a uma grande parcela do mercado de cuidados com a saúde, que cresce rapidamente ${ }^{1}$, tornando-se uma opção comum em tratamentos de Reabilitação Oral em pacientes totais ou parcialmente edêntulos ${ }^{2}$.

O sucesso de um implante depende do contato direto entre osso e implante, denominado osseointegração, onde uma boa ancoragem do implante não apresenta a interposição de tecido mole entre tecido ósseo e implante ${ }^{3}$.

A taxa de sobrevivência de um implante está relacionada a sua estabilidade primária, porém, mesmo sem que haja a estabilidade primária a osseointegração poderá ocorrer promovendo o sucesso dessa integração ${ }^{4,5}$. Outros fatores podem influenciar nessa osseointegração como: o tipo e formato do osso do receptor, o comprimento, diâmetro e posicionamento do implante, tempo de cicatrização óssea assim como o material e tratamento de superfície do implante ${ }^{6,7}$.

Pesquisas buscam por modificações estruturais nos implantes para melhorias da resposta inicial entre hospedeiro/implante podendo promover a redução do tempo de tratamento. Os benefícios clínicos seriam: reabilitações protéticas mais rápidas e um melhor desempenho em regiões de baixa qualidade óssea ${ }^{8,9}$.

Estudos experimentais em animais são necessários para avaliar previamente o resultado de pesquisas e avaliar a necessidade de serem realizadas em humanos. 
É importante a avaliação dos estudos de implante, osso e articulação para compreender os conceitos básicos da estrutura óssea e das vantagens e desvantagens dos diferentes modelos animais. Embora nenhum modelo apresentouse de forma ideal, várias espécies podem fornecer suporte para pesquisas ${ }^{2}$. Os testes em modelos animais clinicamente relevantes são um aspecto importante durante estudos desse tipo e, na maioria dos casos, requisito fundamental para o início da experimentação em humanos ${ }^{10}$. Embora nenhum modelo seja ideal, várias espécies podem fornecer suporte para pesquisas. Muitos modelos animais foram empregados para cicatrização intra e extra oral do tecido ósseo em torno dos implantes dentários, incluindo modelos como ovelhas ${ }^{2}$.

O modelo experimental em ovelhas tem crescido nas pesquisas da implantodontia, sendo utilizado para modelar defeitos em ossos longos, testar materiais em defeitos cranianos, resposta tecidual e abordagens cirúrgicas ${ }^{1,11}$.

Pesquisas recentes demonstram a utilização das ovelhas para pesquisas em implantodontia ${ }^{2,12,13}$, sendo que alguns autores utilizam esses implantes no ângulo da mandíbula desses animais, como o modelo dessa pesquisa ${ }^{14,15,16}$.

Os implantes osseointegrados são clinicamente caracterizados pela ausência de mobilidade, que pode ser avaliada pela medição da estabilidade do implante primário e secundário ${ }^{17}$.

Métodos de testes mecânicos como torque inverso ou "destorque", foram utilizados para estudar e medir essa interface mecânica entre implante e osso ${ }^{3}$. Existem métodos não invasivos que fazem uma estimativa da presença ou ausência de mobilidade óssea por meio da Análise de Frequência de Ressonância (RFA), como o utilizado pelo dispositivo Osstell ${ }^{\mathrm{TM}}$.

A Análise de Freqüência de Ressonância foi utilizada para documentar mudanças na cicatrização óssea ao longo da interface implante-osso, medindo a rigidez do implante no tecido ósseo. Também foi utilizado para determinar se, os implantes estão prontos para a restauração final estando apto para receber uma força de carregamento ou para identificar os implantes em "risco".

Os primeiros estudos com RFA foram publicados no ano de $1996^{18}$.Um ano após, Meredith et al. ${ }^{19}$ sugeriu um método não-invasivo para determinar a freqüência de ressonância associada aos implantes dentários, conectando um adaptador I transdutor na plataforma do implante em um estudo com animais. O sistema RFA experiente, baseado em pulsos magnéticos, começou a ser produzido 
comercialmente três anos mais tarde com com a denominação de Osstell (Osstell AB, Gotemburgo, Suécia). Esse dispositivo calcula o Quociente de Implantação (ISQ) convertendo unidades de kilohertz para ISQ em uma escala de 1 a100, onde quanto maior o valor, maior a estabilidade do implante. Os aumentos nas medidas de ISQ indicam uma maior rigidez óssea e melhor cicatrização ao redor do implante apresentando uma boa estabilidade. O Osstell ISQ funciona através da introdução de uma vibração controlada no implante por meio de um sensor e uma haste (SmartPeg) conectados ao implante medindo sua frequência que 20,21,22.

Esse estudo teve como objetivo avaliar um dispositivo extensor, de seis milímetros de comprimento foi acoplado ao implante, reproduzindo a conexão cônica com precisão, permitindo com isso o acoplamento e utilização de um SmartPeg, padrão Ostell, possibiltando a medição do ISQ nos implantes dentários cone-morse instalados na mandíbula dos animais. A medição do ISQ foi realizada de tal forma que não foi necessária a realização de uma nova cirurgia de exposição do implante, preservando com isso a saúde e integridade do animal, além de facilitar as análises e o manuseio após os períodos de cicatrização.

\section{MATERIAL E MÉTODOS}

Foram utilizados Implantes Cone-Morse Acqua $\AA$ Neodent $($ com tratamento de superfície microtexturizada e propriedade de superfície hidrofílica no o intuito de avaliar e comparar o grau de osseointegração por meio da análise de frequência de ressonância em ovelhas a partir dodesenvolvimento de um dispositivo pela empresa Neodent ${ }^{\circledR}$ (FIG 1 e 2), servindo como uma extensão de $6 \mathrm{~mm}$, permanecendo externo a região do ângulo da mandíbula da ovelha.

Essa extensão permite que o teste mecânico da Análise de Frequência de Ressonância pelo dispositivo Osstell ISQ TM (FIG 3 e 4) seja realizado de forma simplificada, evitando um novo processo cirúrgico, não sendo necessária uma reabertura para se obter acesso aos implantes instalados, além de facilitar sua localização, trazendo benefícios quanto ao manuseio dos animais.

Essa investigação foi desenvolvida pelo grupo de pesquisas do Departamento de Prótese da Faculdade de Odontologia de Bauru (FOB/USP) em parceria com a Faculdade de Zootecnia e Engenharia de Alimentos de Pirassununga (FZEA/USP), 
previamente aprovada pelo Comitê de Ética local em uso animal através pelo protocolo do Centro de Experimentação de Uso Animal (CEUA) nº 5481280616.

\section{Seleção dos animais:}

Foram selecionadas quatro ovelhas, adultas, em bom estado de saúde geral, com idade entre 12 e 24 meses, pesando cerca de $25 \mathrm{~kg}$, passando previamente por uma avaliação de um veterinário da USP de Pirassununga sendo realizado tratamento profilático a infecções e a vacinação. A alimentação foi similar para todos constituindo-se de silagem de milho e pastagem apropriada à idade e peso dos animais com água abundante.

\section{$\checkmark$ Procedimentos Cirúrgicos:}

No período pré-anestésico, os animais permaneceram em jejum por 12 horas, sem restrição hídrica. Os procedimentos experimentais nas ovelhas foram realizados sobre condições rigorosas de esterilização e assepsia. Os animais serão contidos fisicamente para administração da medicação pré-anestésica, a qual será xilazina $0,1 \mathrm{mg} / \mathrm{kg}$.

Em seguida, foram administrados $4 \mathrm{mg} / \mathrm{kg}$ de propopol intravenoso e mantida anestesia inalatória com isoflorano. Durante todas as etapas cirúrgicas do tratamento as ovelhas foram monitoradas por um profissional veterinário, do Departamento de Medicina Veterinária FZEA/USP.

Foi realizada infiltração local de cloridrato de lidocaína (Xilocaína, Merrel Lepetit Farmacêutica e Industrial Ltda, Guarulhos/SP) para fins de vasoconstricção e isquemia no local, com a finalidade de diminuir o sangramento. Foi realizado tricotomia no local e antissepsia com clorexidina $0,12 \%$, seguido de isolamento da área com campos cirúrgicos descartáveis estéreis. Uma incisão de $6 \mathrm{~cm}$ foi realizada na área e o tecido juntamente com o periósteo afastados. Lojas ósseas foram realizadas com motor específico para implantes convencionais, realizada a sua instalação com torque de $20 \mathrm{~N}$, e sutura do local de forma tradicional. Receberam uma dose única de morfina com $0,5 \mathrm{mg} / \mathrm{kg}$ por via intramuscular, logo após a cirurgia

\section{Instalação dos implantes}

Foram instalados 32 implantes, de 3,5 de diâmetro por $8,5 \mathrm{~mm}$ de comprimento Neodent Curitiba,PR-Brasil Acqua ${ }^{\circledR}$ em 4 ovelhas, sendo oito implantes instalados a 
partir do ramo da mandíbula de cada animal, quatro no lado esquerdo e quatro no lado direito. Dessas 4 ovelhas, foram utilizados 1 para o grupo controle, em que foram realizados procedimentos padrões e 3 para o grupo teste.

\section{$\checkmark$ Grupo Controle}

No grupo controle, imediatamente após a instalação dos implantes, foram conectados os SmartPegs e também realizada a medição do ISQ. (FIG 5). Concluindo as medições, foram instalados cicatrizadores com 3,5 mm de altura e a região suturada. (fig 6 e 7) A altura dos cicatrizadores foi determinada,com o objetivo de facilitar a localização dos implantes após sua cicatrização. Após um período de quatro semanas, as ovelhas passaram pelo mesmo protocolo anestésico como descrito, houve a incisão da área operada e exposição dos cicatrizadores instalados, que foram removidos para o acoplamento do SmartPeg e realizada novamente a mensuração do ISQ. (FIG 8)

\section{$\checkmark$ Grupo Teste}

No grupo teste, imediatamente após a instalação dos implantes foram conectados os dispositivos desenvolvidos para o experimento e acoplados os SmartPegs. (FIG 9) para a execução das medições de ISQ. Após a medição foi realizada a sutura mantendo os dispositivos em nível superficial na derme, permitindo rápido um acesso para a etapa posterior do experimento. (FIG 10 e 11). Após quatro semanas de cicatrização, foram realizadas novas mensurações de ISQ, mas sem a necessidade de cirurgia de reabertura, ou seja, os animais permaneceram no seu estado normal de consciência. Obtidas as leituras pelo Osttell foi realizada a Análise Estatística, com $\mathrm{p}<0,05$ para a comparação dos grupos.

\section{$\checkmark$ Avaliação de Frequência de Ressonância}

A avaliação da frequência de ressonância foi realizada cinco vezes em várias incidências, ou seja, na posição incisal, mesial, distal, lingual e vestibular. Logo após a inserção dos implantes, usando o dispositivo Osstell ISQ ${ }^{\mathrm{TM}}$. Os SmartPegs foram acoplados e estabilizados aos dispositivos personalizados tendo o cuidado de garantir que nenhuma força de torque significativa fosse aplicada aos implantes e a RFA fosse realizado. 


\section{$\checkmark$ Pós-operatório}

Logo após toda e qualquer cirurgia realizada, os animais foram mantidos em alojamento por uma semana para avaliação do seu estado de saúde. Durante os primeiros 7 dias após a cirurgia, realizou-se a administração de anti-inflamatório e analgésico via intramuscular de Meloxicam na dose de $0,1 \mathrm{mg} / \mathrm{kg}$, visando atenuar ao máximo a dor e o edema na área operada, sendo a alimentação retomada após $24 \mathrm{~h}$ dos procedimentos cirúrgicos efetuados. Foi mantida uma observação dos animais quanto às áreas operadas, focando a manutenção das suturas, manutenção da higienização dos implantes e aspectos gerais de saúde dos animais.

\section{Análise Estatística:}

As medidas de RFA foram realizadas em 5 locais em torno de cada implante: mesial, lingual, distal, vestibular e incisal.calculando-se o valor médio desses cinco. A análise estatística para pares equivalentes do teste de Wilcoxon e o teste de Mann Whitney efetuados com um nível de significância de $p<0,05$. 23. A analise estatística foi realizada com o auxílio do software Bioestat. ${ }^{24}$

\section{RESULTADOS}

As médias obtidas no GC foram 50,47 imediatamente após cirurgia e 65,68 após 4 semana, enquanto que no GT foram 34,74 e 34,81, respectivamente. Comparando a diferença entre os grupos nas médias de ISQ imediatas e após quatro semanas, houve diferença significativa com $p=0.0321$ e $p=0.0027$, respectivamente, demonstrando que os resultados no GT nos dois períodos não foram equivalentes quando comparados ao GC.

Os valores médios de ISQ imediatamente após inserção e após 4 semanas foram 50,47 e 65,68 para o Grupo Controle e 34,54 e 34,81 para o Grupo Teste, respectivamente, demonstrados pelas tabelas 1 e 2 .

Os valores ISQ do GC aumentou significativamente considerando a partir do momento imediato da inserção a após quatro semanas de cicatrização com o $p=$ 0.0280. Esse aumento não foi observado nas ovelhas do GT, com o $p=0.7532$ de acordo com a análise de Wilcoxon. 
No comparativo imediato entre grupos, houve diferença significativa nas médias de ISQ entre implantes imediatos Ovelha Controle X Médias Ovelhas teste com o valor de $p=0.0321(p<0.05)$.

Quando comparado o GC com GT no segundo momento, ou seja, após 4 semanas, foi demonstrado que também houve diferença significativa, com valor de $p=0.0027$.

Em geral, os valores das médias entre os grupos demonstraram menores valores no GT em relação ao GC.

A imagem (FIG 12) demonstra os resultados obtidos dos comparativos em um gráfico Boxplot.

No total de 32 implantes instalados, 5 deles não apresentaram osseointegração após quatro semanas, sendo 4 no grupo teste e 1 no grupo controle. Esses implantes apresentaram mobilidade, o que corresponde a uma uma taxa de $15,62 \%$ de insucesso de osseointegraçao dos implantes nesse estudo.

\section{DISCUSSÃO}

A escolha cuidadosa do modelo animal vai depender do objetivo da pesquisa, capacidades pessoais e institucionais, questões éticas e econômicas, além de preferência e experiência dos pesquisadores. Ovelhas são animais grandes e adequados para pesquisa médica devido a disponibilidade e aceitação pela sociedade de pesquisa animal ${ }^{1,25,26,27,}$.

No caso deste estudo, as ovelhas foram os animais escolhidos devido serem animais compatíveis com os humanos em dimensões, peso e fisiologia em geral, assim como por serem de fácil manipulação, boa recuperação após administração de anestesia e após cirurgias. Comparado a fisiologia de outros animais quanto a um defeito ósseo no crânio, podem ser comparados aos cães, macacos e seres humanos ${ }^{2,14,25}$.

Os dados de uma pesquisa são úteis para aplicação clínica em seres humanos baseados no bom planejamento, desenho experimental e também no conhecimento adequado de cada animal, levando em consideração as diferenças com os seres humanos. Devendo ter o foco na preservação animal e o bem-estar em todas as etapas do experimento ${ }^{27}$. A respeito da preservação, que é um assunto 
polêmico em quanto as questões éticas de pesquisa em animais, foi relatado o princípio dos 3Rs: reduzir o número de animais utilizados durante a experimentação, minimizar a dor e desconforto e evitar a eutanásia no final da experimentação 28 . (NC3RS 2010). Levando em consideração para que pesquisas futuras evitem o uso excessivo de animais, evitando também o desperdício de tempo, esforço e recursos, houve uma adaptação nessa pesquisa para o bem-estar do animal, buscando alternativas para permitam que esses animais não sofram eutanásia ${ }^{27,28}$. Os animais adquiridos nessa pesquisa, fazem parte da Faculdade de Zootecnia e Engenharia de Alimentos da USP, e seriam destinados ao abate para produção de carne na área alimentícia, sendo selecionados para reduzir o desperdício do uso animal. Porém, em todas as fases da pesquisa os animais foram mantidos sem realização da eutanásia.

Foram realizados cinco procedimentos cirúrgicos para avaliação do desempenho entre o osso e implantes dentários utilizando um novo dispositivo que possibilitasse a medição da estabilidade óssea. No grupo controle, para acessar a região, foi necessário um novo procedimento cirúrgico, em que a ovelha passaria novamente pelos mesmos procedimentos anestésicos, a administração de drogas, bem como um novo dano no local da cirurgia. Devido à complexidade dessa nova etapa cirúrgica, tanto no âmbito da equipe de pesquisa, dos recursos e também os danos ao animal, desenvolveu-se um dispositivo que facilitasse as investigações.

No grupo teste, a medição do ISQ de implantes dentários instalados na mandíbula de ovelhas foi realizada de tal forma que possibilitasse a avaliação póscirúrgica imediata e após 4 semanas sem a necessidade de uma nova cirurgia de reabertura, e futuramente, novas análises em mais períodos, sendo que seu uso não apresentou intercorrências, facilitando o manuseio animal sem a necessidade de exposição cirúrgica num segundo estágio.

As mandíbulas de ovelhas foram usadas para determinar mudanças longitudinais na freqüência de ressonância e medidas imediatamente após a instalação dos implantes e após 4 semanas, e observando que os valores de RFA aumentaram ao longo desse tempo no grupo controle, o que não foi observado no grupo teste.

São demonstradas outras investigações com implantes na mandíbula de ovelhas $^{13,17,18,33}$ seja no ângulo da mandíbula ou na sua borda inferior, demonstrando efetividade na compreensão dos processos de cicatrização óssea e 
testes biomecânicos sobre os implantes dentários. Estudos semelhantes também são demonstrados utilizando a crista ilíaca para ancoragem de implantes ${ }^{25,29}$.

Alguns estudos mostraram que os valores de ISQ aumentam com o tempo após a colocação do implante atingindo um pico entre 6 e 8 semanas. Isso pode estar relacionado ao aumento da rigidez de um implante nos tecidos circundantes que ocorre durante a fase de cicatrização ${ }^{30}$.

Sabe-se que o ISQ>70 corresponde alta estabilidade, entre 60 e 69 média estabilidade e ISQ $<60$ corresponde a baixa estabilidade de acordo com mais de 700 referências, sendo uma ferramenta que substituiu progressivamente outros métodos na pesquisa na Implantodontia ${ }^{23}$.

E outra investigação já demonstra que todos os implantes apresentaram níveis favoráveis de RFA que foram encontrados na terceira semana ${ }^{31}$.

Em contrapartida, outros estudos não demostraram diferenças significativas como uma investigação ${ }^{17}$, em que foram instalados implantes no ângulo da mandíbula e feitos testes RFA em vários períodos, sendo que um deles após 4 semanas, não apresentando diferença estatística nos períodos observados.

Alguns resultados diversos mostraram diminuição do valor de RFA durante as primeiras 2 semanas após a colocação do implante, e após esse período um aumento na estabilidade. Esta alteração pode estar relacionada à cicatrização óssea precoce, como mudança biológica e reabsorção óssea alveolar marginal. $\mathrm{O}$ remodelamento ósseo reduz o contato primário do osso e no estágio inicial após a colocação do implante, a formação de calo ósseo e o aumento do osso lamelar no osso cortical causam grandes alterações na densidade óssea. Assim, no processo de cicatrização, o contato ósseo primário diminui e o contato ósseo secundário aumenta $^{32,33,34} 35,36,37$

Com base nas considerações acima, sugere-se que métodos quantitativos de avaliação da estabilidade do implante em humanos sejam complementares de outros métodos de análise. No caso da utilização dos implantes para reabilitações de pacientes, os exames clínicos e radiográficos também devem ser realizados, procurando características como falta de mobilidade, ausência de dor, ausência de infecção recorrente e ausência de radiolucência contínua ao redor do implante após 3, 6 e 12 meses de carregamento, que são considerados critérios que definem o sucesso de um implante ${ }^{10,38,39 .}$ 
Além disso, outros estudos devem distinguir diferentes situações clínicas, tais como carregamento imediato / tardio e locais de implantação pós-extração / cicatrizados, já que esse método pode ser usado no momento da inserção do implante, durante o período de cicatrização e quando a prótese dentária está em função. ${ }^{40}$

Sobre a diferença de valores de ISQ encontrados, um estudo demonstrou a relação entre os valores ISQ entre diferentes pilares protéticos e com a plataforma do implante. Os transdutores SmartPegs foram fixos aos componentes ou à plataforma do implante e todos os valores nos pilares eram inferiores aos da plataforma do implante, para os mesmos implantes. Os resultados deste estudo sugerem que os valores ISQ obtidos no pilar não podem ser comparados com os valores da plataforma. Esse fenômeno foi explicado pois os transdutores (SmartPegs) imprimem uma força lateral no componente protético quando a medição é realizada, sendo o deslocamento maior, pois aumenta o braço da alavanca em relação à medida feita diretamente na plataforma. Assim, quando a RFA é medida sobre um componente, um valor mais baixo é esperado para o mesmo implante, em comparação com o valor medido diretamente na sua plataforma ${ }^{41}$.

Esses autores sugerem que estudos adicionais devem ser realizados com diferentes diâmetros e conexão do implante, e diferentes componentes para confirmar os achados presentes. Portanto, a altura do componente sobre a plataforma do implante teve um impacto significativo nas medidas de RFA, quanto maior a altura do componente, menor o valor do quociente de estabilidade do implante. Esses resultados estão de acordo com os resultados obtidos, em que os valores de RFA foram diferentes no momento da instalação dos implantes e após 4 semanas, quando comparamos o acoplamento do Smartpeg diretamente na plataforma do implante e no componente personalizado.

Se o implante não estiver suficientemente estabilizado no momento da colocação, uma cápsula de tecido fibroso pode formar em vez de osso, o que pode levar à falha de osseointegração e posterior falha no implante ${ }^{25}$. O que pode ser explicado no caso das falhas de implantes que obtivemos nesse estudo, sendo que de 32 implantes instalados no total, 5 deles apresentaram falhas após 4 semanas. No entanto, trata-se de um estudo experimental realizado em um modelo animal, 
que envolve uma série de limitações, como a qualidade do osso em uma área diferente da cavidade oral e com um número de amostras reduzido.

Uma outra investigação demonstrou a incorporação de um implante em um bloco de alumínio com alturas diferentes e a obtenção do valor RFA pareceu mudar de acordo com essas alturas, sugerindo uma forte correlação $(r=0,94, p<0,01)$ entre a freqüência observada e a altura da fixação do implante exposta21.

De acordo com a literatura, pesquisas demonstram que o sistema RFA contribuído por Osstell ${ }^{\mathrm{TM}}$ contribui com medidas RFA altamente confiáveis em implantes dentários ${ }^{42}$.

Em outro estudo, desenvolveu-se um SmartPeg customizado para realizar medidas de RFA em mini-implantes, demonstrando que desenvolvimento de métodos adicionais são efetivos para a busca de uma melhor compressão dos processos biológicos ${ }^{43}$.

De acordo com os resultados e com a literatura, o uso de componentes personalizados não demonstrou total efetividade quando comparados com o padrão original de medição, ou seja, quando o acoplamento do transdutor SmartPeg ocorre diretamente na plataforma do implante.

\section{CONCLUSÃO}

Após os resultados encontrados, podemos chegar as seguintes conclusões:

A avaliação do componente apresentou resultados satisfatórios no quesito do bem-estar animal e economia de recursos, mas os resultados demonstram que apesar de seus benefícios para a pesquisa, não demonstrou equivalência nos resultados nas medições de ISQ quando comparados ao método convencional.

Novos dispositivos e materiais devem ser desenvolvidos e analisados com a finalidade de simplificar estudos científicos em animais. 


\section{REFERÊNCIAS}

1. Wancket LM. Animal Models for Evaluation of Bone Implants and Devices. Veterinary Pathology Vol 52, Issue 5, pp. 842 - 850 First published date: July10-2015

2. Duncan W, Lee M, Bae T, Lee S, Gay J, Loch C. Anodisation Increases Integration of Unloaded Titanium Implants in Sheep Mandible. BioMed Research International Vol. 2015.

3. Branemark R, Ohrnell LO, Nilsson P, Thomsen P. Biomechanical characterization of osseointegration during healing: an experimental in vivo study in the rat. Biomaterials. 1997;18(14):969-78. doi: 10.1016/S01429612(97)00018-5.

4. Yurttutan ME, Kestane R, Keskin A, Dereci O. Biomechanical evaluation of oversized drilling on implant stability - an experimental study in sheep. J Pak Med Assoc Vol. 66, No. 2, February 2016

5. Jung UW, Kim S, Kim YH, Cha JK, Lee IS, Choi SH. Osseointegration of dental implants installed without mechanical engagement: a histometric analysis in dogs. ClinOral Implants Res. 2012; 23:1297-301

6. Shalabi MM, Gortemaker A, Van't hof MA, Jasen JA, Reugers NHJ. Implant surface roughness and bone healing: a systematic review. J Dent Res 2006;85:496-500.

7. Mesa F, Munõs R, Noguerol B, De Dios Luna J, Galindo P, O'valle F. Multivariate study of factors influencing primary dental implant stability. Clin Oral Implants Res 2008;19:196-200.

8. Buser D, Mericske-Stern R, Bernard JP, et al. Long-term evaluation of nonsubmerged ITI implants. Part 1: 8-year life table analysis of a prospective multicenter study with 2359 implants. Clin Oral Implants Res 1997;8:161-172.

9. Sartoretto SC, Alves ATNN, Zarranz L, Jorge MZ, Granjeiro JM, CalasansMaia MD. Hydrophilic surface of Ti6Al4V-ELI alloy improves the early bone apposition of sheep tibia. Clin. Oral Impl. Res. 00, 2016, 1-9 doi: 10.1111/clr.12894

10. Bowers M, Yoo D, Marin C, Gil L, Shabaka N, Goldstein M, Janal M, Tovar N, Hirata R, Bonfante E, Coelho P. Surface characterization and in vivo evaluation of laser sintered and machined implants followed by resorbable-blasting media process: A study in sheep. Med Oral Patol Oral Cir Bucal. 2016 Mar 1;21 (2):e206-13.

11. Duncan, WJ. Sheep mandibular animal models dental implantology research. A thesis submitted for the degree of Doctor of Philosophy at the University of Otago, Dunedin, New Zealand, Jan. 2005.

12. Yoo D, Marin C, Freitas G, Tovar N, Bonfante E, Teixeira H, Janal M, Coelho P. Surface Characterization and In Vivo Evaluation of Dual Acid-Etched and GritBlasted/Acid-Etched Implants in Sheep. Implant Dentistry. Vol. 24 N. 3, 2015. 
13. Ernst S, Stubinguer S, Schupbach P, Sidler M, Klein K., Ferguson S, Rechenberg B. Comparison of two dental implant surface modifications on implants with same macrodesign: an experimental study in the pelvic sheep model. Clin. Oral Impl. Res. 26, 2015, 898-908.

14. Trisi $P$, Todisco M, Consolo $U$, Travaglini, D. High versus low implant insertion torque: a histologic, histomorphometric, and biomechanical study in the sheep mandible. Int J Oral Maxillofac Implants. 2011 Jul-Aug;26(4):837-49.

15. Dagher M, Mokbel N, Jabbour G, Naaman N. Resonance Frequency Analysis, Insertion Torque, and Bone to Implant Contact of 4 Implant Surfaces: Comparison and Correlation Study in Sheep. Implant Dentistry. 2014 vol. 23 n.6.

16. Xiao J, Li D, Chen Y, Chen N S, Kong, L. Evaluation of Fixation of Expandable Implants in the Mandibles of Ovariectomized Sheep. J Oral Maxillofac Surg 71:682-688, 2013.

17. Atsumi M, Park SH, Wang HL. Methods used to assess implant stability: current status. Int J Oral Maxillofac Implants. 2007;22(5):743-54.

18. Meredith N, Alleyne D, Cawley P. Quantitative determination of the stability of the implant-tissue interface using resonance frequency analysis. Clin Oral Implants Res. 1996;7(3):261-7. doi: 10.1034/j.1600-0501.1996.070308.x.

19. Meredith N, Shagaldi F, Alleyne D, Sennerby L, Cawley P. The application of resonance frequency measurements to study the stability of titanium implants during healing in the rabbit tibia. Clin Oral Implants Res. 1997;8(3):234-43. doi: 10.1034/j.1600-0501.1997.080310.x

20. Bischof M, Nedir R, Szmukler-Moncler S, Bernard JP, Samson J. Implant stability measurement of delayed and immediately loaded implants during healing. Clin Oral Implants Res. 2004;15:529-539.

21. Alsaadi G, Quirynen M, Michiels K, Jacobs R, van Steenberghe D. A biomechanical assessment of the relation between the oral implant stability at insertion and subjective bone quality assessment. $\mathrm{J}$ Clin Periodontol 2007;34:359-366.

22. Santamaría-Arrieta, G, Brizuela-Velasco A, Fernández-González F, ChávarriPrado D, Chento-Valiente Y, Solaberrieta E, Yurrebaso-Asúa, J. (2016). Biomechanical evaluation of oversized drilling technique on primary implant stability measured by insertion torque and resonance frequency analysis. Journal of Clinical and Experimental Dentistry, 8(3), e307-e311. http://doi.org/10.4317/jced.52873

23. Vieira S. Bioestatística: tópicos avançados. Rio de Janeiro: Elsevier, 2004.

24. Ayres M, Ayres Júnior M, Ayres DL, SANTOS AA. 2007. BIOESTAT Aplicações estatísticas nas áreas das ciências bio-médicas. Ong Mamiraua. Belém, PA.

25. Scarano A, Lorusso F, Ravera L, Mortellaro C, Piattelli A. Bone Regeneration in Iliac Crestal Defects: An Experimental Study on Sheep. BioMed Research International. 2016;2016:4086870. doi:10.1155/2016/4086870. 
26. Alayan J, Vaquette C, Saifzadeh S, Hutmacher D, Ivanovski S. Comparison of early osseointegration of SLA and SLActive implants in maxillary sinus augmentation: a pilot study. Clin. Oral Impl. Res. 00, 2016, 1-9 doi: $10.1111 / \mathrm{clr} .12988$

27. Turner AS. Experiences with sheep as an animal model for shoulder surgery Strengths and shortcomings. J Shoulder Elbow Surg. 2007;16(5):158S-163S.

28. NC3Rs Reporting Guidelines Working Group (2010) Animal research: reporting in vivo experiments: the ARRIVE guidelines. The Journal of Physiology 588: 2519-2521.

29. Trisi P, Berardini M, Falco A, Podaliri Vulpiani M. Effect of Implant Thread Geometry on Secondary Stability, Bone Density, and Bone-to-ImplantContact: A Biomechanical and Histological Analysis. Implant Dent. 2015 Aug;24(4):38491. doi: 10.1097/ID.0000000000000269

30. Trisi P, Berardini M, Falco A, Sandrini E, Vulpiani MP. A New Highly Hydrophilic Electrochemical Implant Titanium Surface: A Histological and Biomechanical In Vivo Study. Implant Dent. 2017 Jun;26(3):429-437. doi: 10.1097/ID.0000000000000605.

31. Huang HM, Chiu CL, Lin CT, Lin LH, Lee SY. Early detection of implant healing process using resonance frequency analysis. Clin Oral Implants Res. 2003;14:437-443).

32. Abdel-Haq, J., Karabuda, CZ, Arısan, V., Mutlu, Z. e Kürkçü, M. Osseointegração e estabilidade de um implante de ácido acidificado: um estudo piloto experimental em ovelhas. Clinical Oral Implants Research, 22: 265-274, 2011 doi: 10.1111 / j.1600-0501.2010.01990.x

33. Satwalekar, P., Nalla, S., Reddy, R., \& Chowdary, S. G. (2015). Clinical evaluation of osseointegration using resonance frequency analysis. The Journal of the Indian Prosthodontic Society, 15(3), 192-199. http://doi.org/10.4103/0972-4052.165171

34. Perrotti V, Piattelli A, lezzi G. Mineralized bone-implant contact and implant stability quotient in 16 human implants retrieved after early healing periods: A histologic and histomorphometric evaluation. Int $J$ Oral Maxillofac Implants. 2010;25:45-8.)

35. Sim, CPC, Lang NP. Factors influencing resonance frequency analysis assessed by Osstell ${ }^{\mathrm{TM}}$ mentorduring implant tissue integration: I. Instrument positioning, bone structure, implant length. Clinical Oral Implants Research 2010, 21: 598-604. doi:10.1111/j.1600-0501.2009.01878.x).

36. Salata LA., Burgos PM, Rasmusson L, et al. Osseointegration of oxidized and turned implants in circumferential bone defects with and without adjunctive therapies: an experimental study on BMP-2 and autogenous bone graft in the dog mandible. International Journal of Oral and Maxillofacial Surgery. 2007;36(1):62-71. doi: 10.1016/j.ijom.2006.07.009.

37. Sul YT, Jönsson J, Yoon GS, Johansson C. Resonance frequency measurements in vivo and related surface properties of magnesiumincorporated, micropatterned and magnesium-incorporated TiUnite, Osseotite, 
SLA and TiOblast implants. Clinical Oral Implants Research. 2009;20(10):1146-1155. doi: 10.1111/j.1600-0501.2009.01767.x.

38. Zix J, Hug S, Kessler-Liechti G, Mericske-Stern R. Measurement of dental implant stability by resonance frequency analysis and damping capacity assessment: Comparison of both techniques in a clinical trial. Int $J$ Oral Maxillofac Implants 2008;23:525-530.

39. Andreotti AM, Goiato MC, Nobrega AS, et al. Relationship between implant stability measurements obtained by two different devices: a systematic review. J Periodontol. 2017;88(3):281-288.

40. Lages FS, Douglas-de Oliveira DW, Costa FO. Relationship between implant stability measurements obtained by insertion torque and resonance frequency analysis: A systematic review. Clin Implant Dent Relat Res. 2018;20:26-33. https://doi.org/10.1111/cid.12565).

41. Lages, FS, Willya Douglas-de-Oliveira D, Ibelli, GS, Assaf F, Queiroz TP, Costa FO. (2017). Relationship between implant stability on the abutment and platform level by means of resonance frequency analysis: A cross-sectional study. PLoS ONE, $12(7), \quad$ e0181873. http://doi.org/10.1371/journal.pone.0181873)

42. Herrero-Climent, M, Albertini M, Rios-Santos JV, Lázaro-Calvo P, FernándezPalacín A, Bullon P. (2012). Resonance frequency analysis-reliability in third generation instruments: Osstell mentor®. Medicina Oral, Patología Oral Y Cirugía Bucal, 17(5), e801-e806. http://doi.org/10.4317/medoral.17861.

43. Dhaliwal JS, Albuquerque RF, Fakhry A, Kaur S, Feine JS. Customized SmartPeg for measurement of resonance frequency of mini dental implants. International Journal of Implant Dentistry. 2017;3:4. doi:10.1186/s40729-017-0066-6. 


\section{LEGENDAS PARA ILUSTRAÇÕES}

Figura 1 - Desenho para fabricação do componente para implantes cone morse Figura 2 - Componente após sua fabricação e antes de ser instalado sobre a plataforma do implante cone morse

Figura 3 - Dispositivo Osstell ISQ ${ }^{\mathrm{TM}}$

Figura 4 - Demonstração do acoplamento do SmartPeg ao Componente de 6mm Figura 5 - Instalação do SmartPeg à plataforma do implante e obtenção do ISQ pelo transdutor Osstell ${ }^{\mathrm{TM}}$

Figura 6 - Instalação de cicatrizadores de 3,5mm sobre a plataforma dos implantes cone morse

Figura 7 - Aspecto após sutura na Ovelha Controle

Figura 8 - Medição do ISQ na Ovelha Controle, após reabertura dos implantes, remoção dos cicatrizadores e acoplamento do SmartPeg

Figura 9 - Instalação do componente após instalação dos implantes no Grupo Teste

Figura 10 - Aspecto após sutura, demonstrando a extensão externa dos componentes numa vista aproximada

Figura 11 - Aspecto após sutura, demonstrando a extensão dos componentes numa visão ampliada, demonstrando a região do ângulo da mandíbula da ovelha Figura 12 - Box-plot geral, demonstrando médias de ISQ dos grupos Teste e Controle imediatamente após instalação dos implantes e após 4 semanas 
Fig. 1

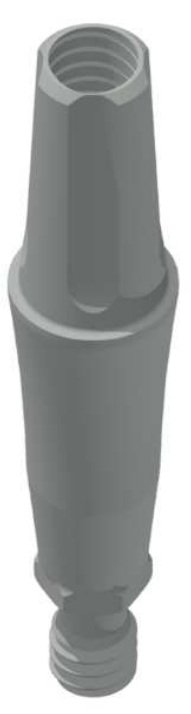

Fig. 2

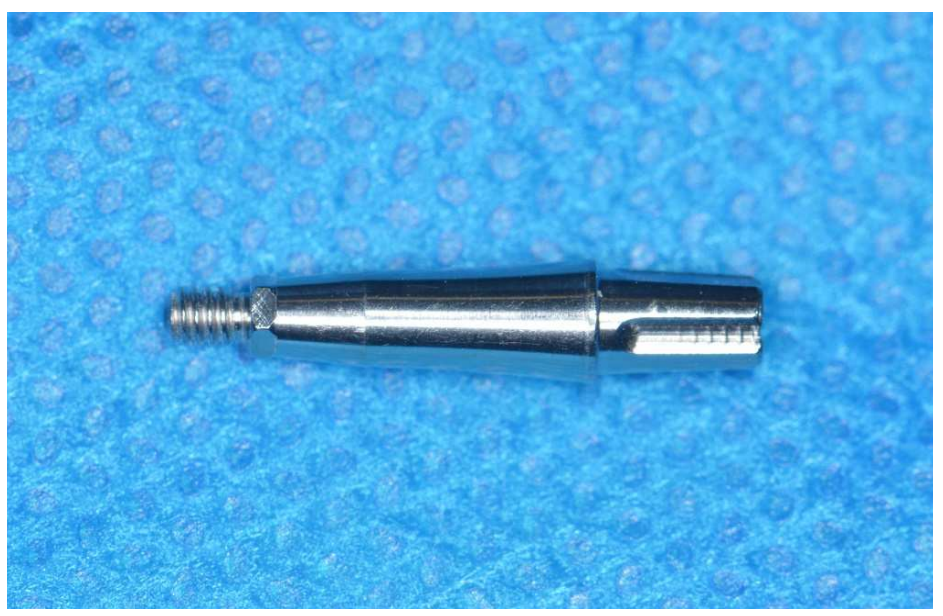


Fig. 3

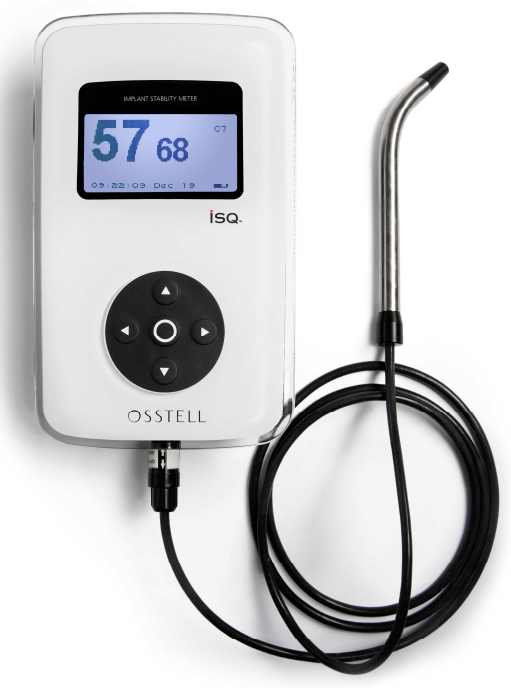

Fig. 4

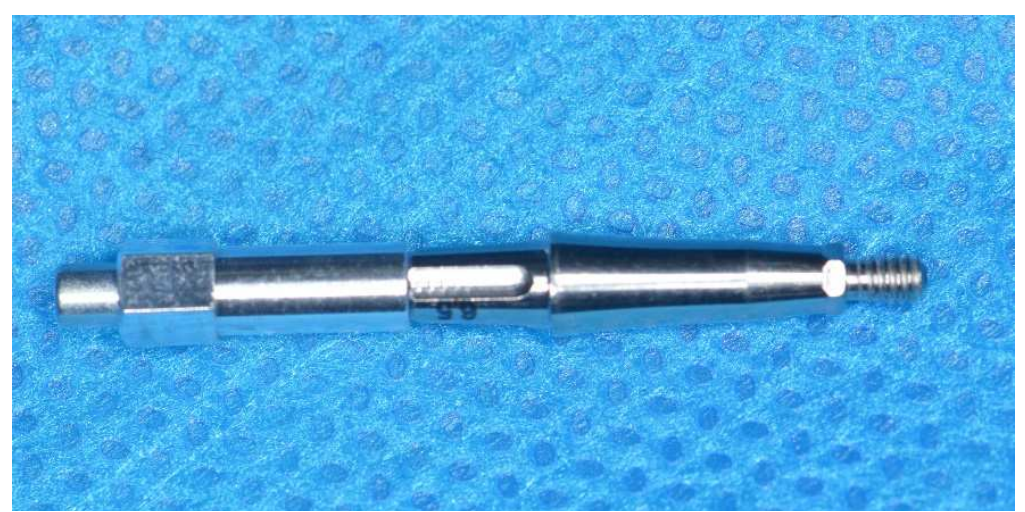


Fig. 5

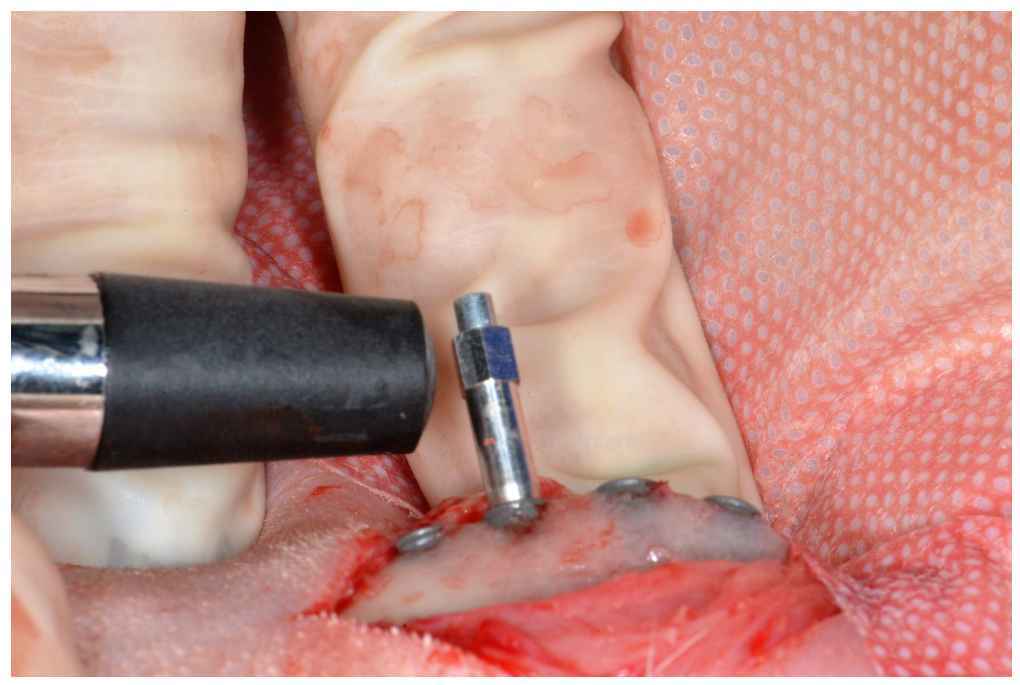

Fig. 6

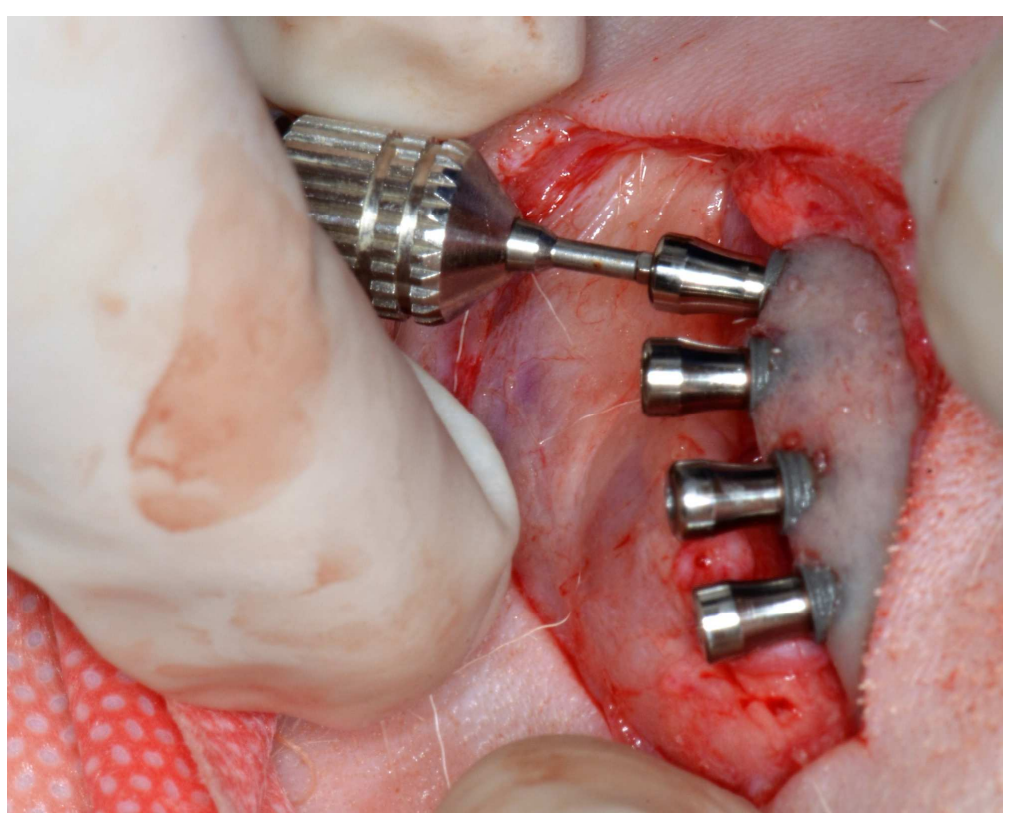


Fig. 7

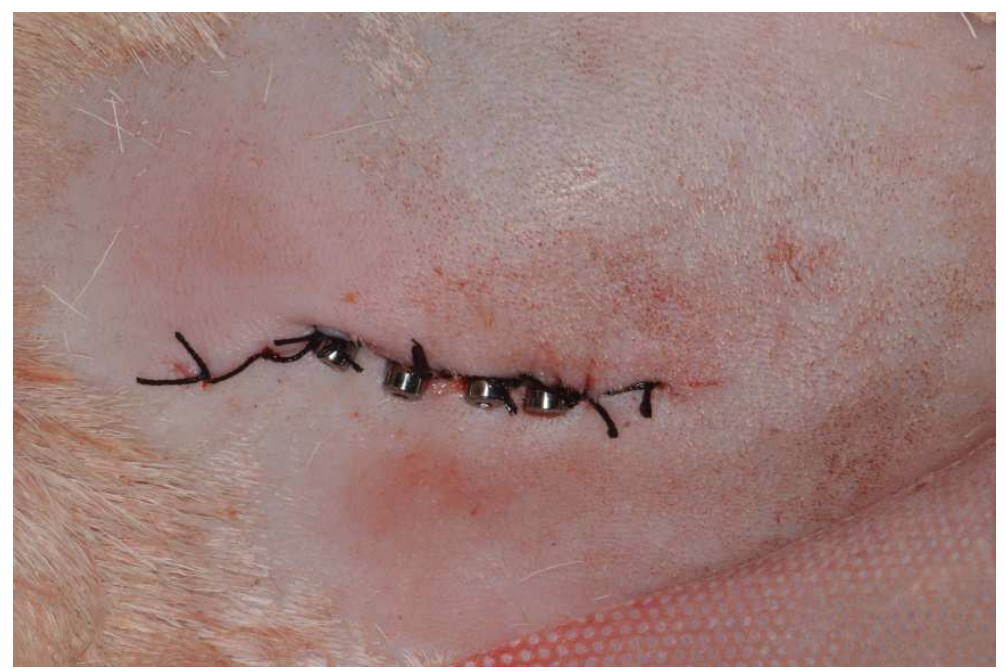

Fig. 8

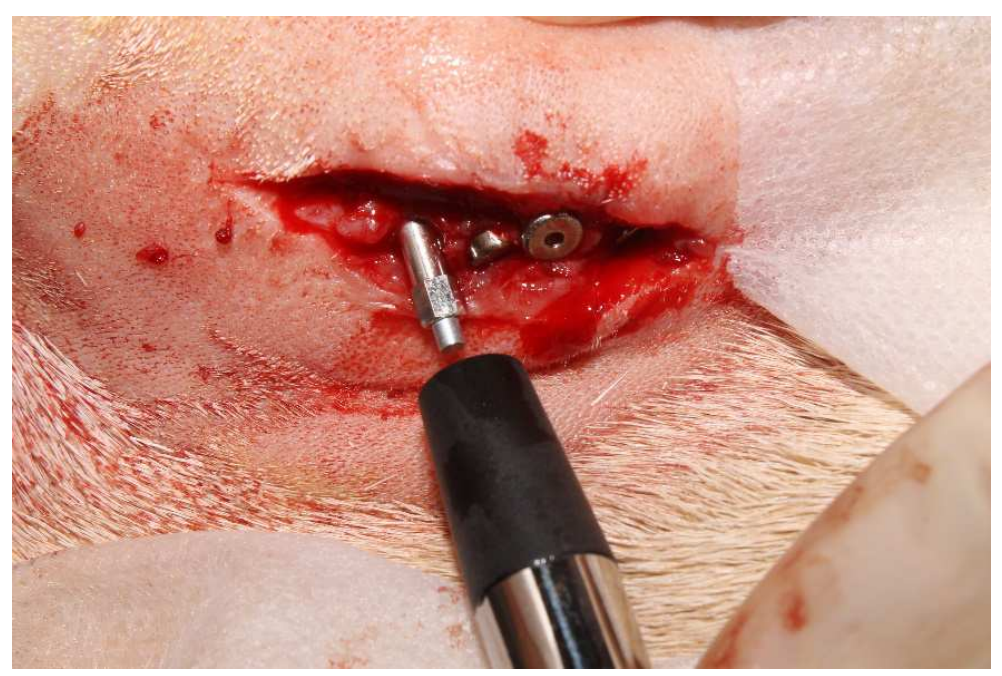

Fig. 9

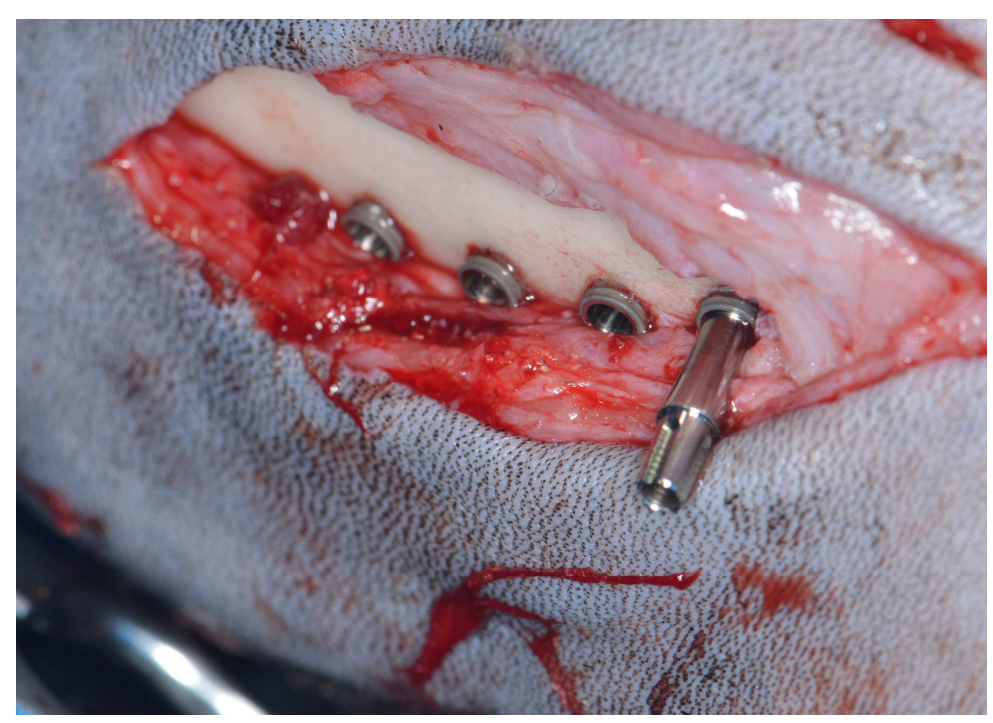


Fig. 10

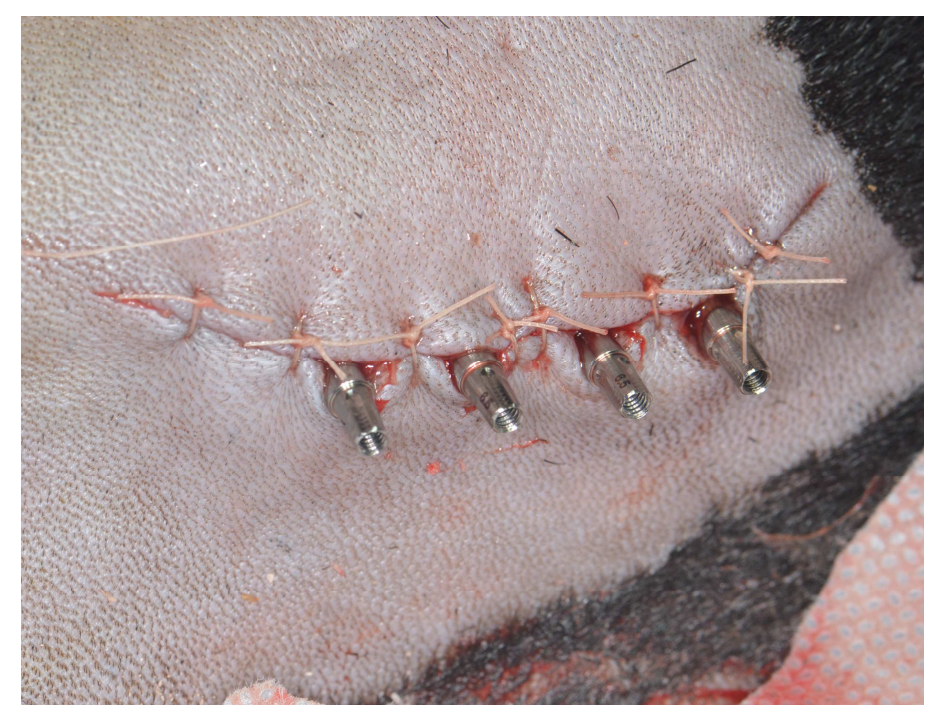

Fig. 11

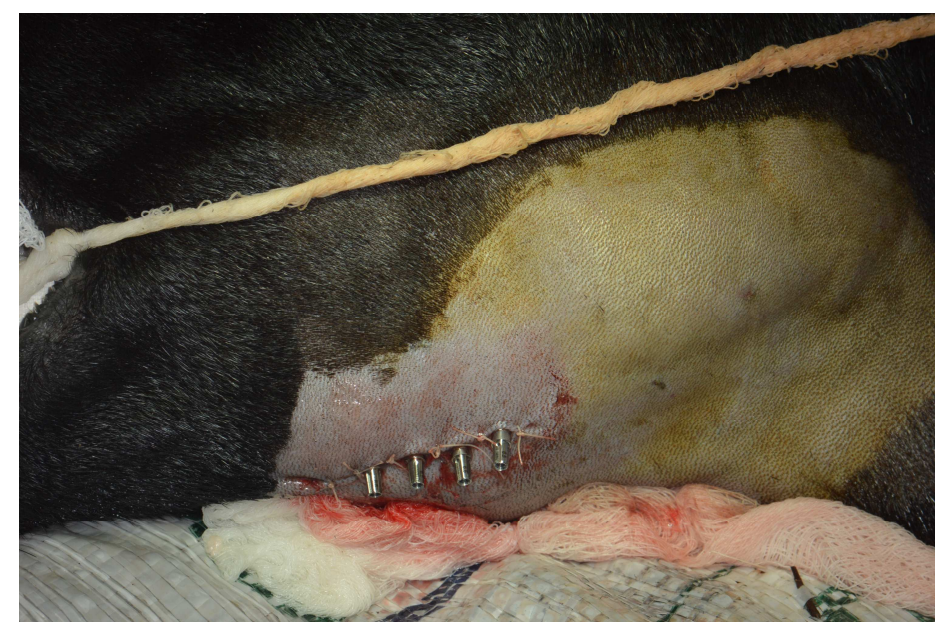


Fig. 12

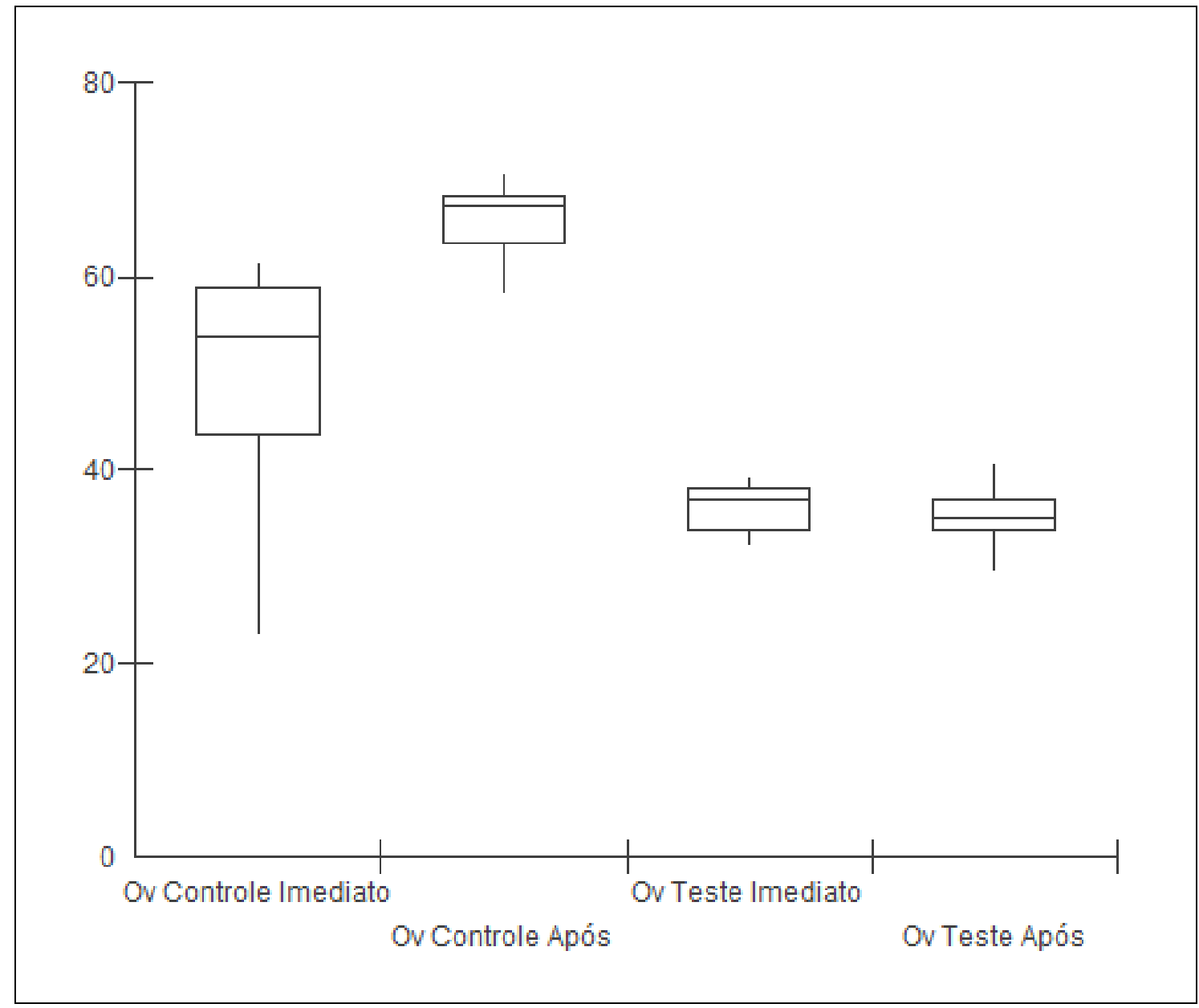




\section{LEGENDAS PARA TABELAS}

Tabela 1. Valores médios de ISQ no grupo Controle imediatamente após instalação dos implantes e após 4 semanas

Tabela 2. Valores médios de ISQ no grupo Teste imediatamente após instalação dos implantes e após 4 semanas

Tabela 1: Médias ISQ, ovelha controle.

\begin{tabular}{l|crc}
\hline & & \multicolumn{2}{c}{ Após 4 } \\
\hline Lado Esquerdo & Implantes & Imediato & semanas \\
& I & 61.20 & 58.20 \\
& II & 60.80 & perdido \\
& III & 56.80 & 67.40 \\
Lado Direito & IV & 60.80 & 68.80 \\
& & & \\
& I & 53.80 & 62.80 \\
& II & 47.60 & 67.80 \\
& III & 39.80 & 64.20 \\
& IV & 23.00 & 70.60 \\
\hline
\end{tabular}

Tabela 2: Médias ISQ, ovelhas teste.

\begin{tabular}{|c|c|c|c|}
\hline & Implantes & Imediato & $\begin{array}{l}\text { Após } 4 \\
\text { semanas }\end{array}$ \\
\hline Lado & & & \\
\hline \multirow[t]{4}{*}{ Esquerdo } & I & 33.00 & 29.53 \\
\hline & II & 37.40 & 34.07 \\
\hline & III & 32.27 & 37.40 \\
\hline & IV & 38.47 & 35.80 \\
\hline \multirow[t]{4}{*}{ Lado Direito } & I & & nd \\
\hline & II & 39.13 & 33.60 \\
\hline & III & 36.33 & 40.60 \\
\hline & IV & 36.60 & nd \\
\hline
\end{tabular}



3 Discussão 



\section{DISCUSSÃO}

A escolha cuidadosa do modelo animal vai depender do objetivo da pesquisa, capacidades pessoais e institucionais, questões éticas e econômicas, além de preferência e experiência dos pesquisadores. Ovelhas são animais grandes e adequados para pesquisa médica devido a disponibilidade e aceitação pela sociedade de pesquisa animal. Possuem semelhança com os seres humanos no peso, estrutura e regeneração óssea 1,26,27,28.

No caso deste estudo, as ovelhas foram os animais escolhidos devido serem animais compatíveis com os humanos em dimensões, peso e fisiologia em geral, assim como por serem de fácil manipulação, boa recuperação após administração de anestesia e após cirurgias. Comparado a fisiologia de outros animais quanto a um defeito ósseo no crânio, podem ser comparados aos cães, macacos e seres humanos $2,14,29$.

A literatura é limitada em relação aos protocolos de sistemas de biomateriais e implantes em comparação com animais de pequeno porte, como os roedores².

Os dados de uma pesquisa são úteis para aplicação clínica em seres humanos baseados no bom planejamento, desenho experimental e também no conhecimento adequado de cada animal, levando em consideração as diferenças com os seres humanos. Devendo ter o foco na preservação animal e o bem-estar em todas as etapas do experimento ${ }^{30}$.

Deve-se tomar cuidado de usar o mesmo gênero de animais em cada experimento para evitar vieses. Nesse estudo todos os animais utilizados eram fêmeas e estavam em boa saúde durante o período cirúrgico, apresentando uma boa recuperação após suspensão da anestesia.

A respeito da preservação, que é um assunto polêmico em quanto as questões éticas de pesquisa em animais, foi relatado o princípio dos 3Rs: reduzir o número de animais utilizados durante a experimentação, minimizar a dor e desconforto e evitar a eutanásia no final da experimentação ${ }^{31}$. Levando em consideração para que pesquisas futuras evitem o uso excessivo de animais, 
evitando também o desperdício de tempo, esforço e recursos, houve uma adaptação nessa pesquisa para o bem-estar do animal, buscando alternativas para o melhor conforto, permitindo que que esses animais não sofressem eutanásia ${ }^{30,31}$. Os animais adquiridos nessa pesquisa, fazem parte da Faculdade de Zootecnia e Engenharia de Alimentos da USP, e seriam destinados ao abate para produção de carne na área alimentícia. Então foram selecionados, para reduzir o desperdício do uso animal. Porém, em todas as fases da pesquisa os animais foram mantidos sem realização da eutanásia.

Esse estudo propõe a utilização de ovelhas como alternativa em pesquisas in vivo na área de Implantodontia e na busca de novos biomateriais, relatando as experiências adquiridas para servir como base para novos estudos, além de demonstrar a experiência com a utilização de um componente personalizado para ancoragem ao SmartPeg do sistema Osstel ISQ ${ }^{\text {TM }}$ e obtenção de valores de RFA através da escala ISQ, demonstrando a estabilidade óssea desses implantes após o período de cicatrização.

No método RFA, um sensor "Smartpeg" é acoplado ao implante, e subsequentemente a ponta do dispositivo é mantida próxima ao sensor nas direções vestibular, lingual, mesial, distal e incisal durante o período de emissão de impulsos eletromagnéticos, medindo a rigidez do implante, determinando sua estabilidade óssea. Após esse período, os valores de freqüência de ressonância são automaticamente convertidos em uma escala arbitrária chamada quociente de estabilidade do implante (ISQ) e mostrada na exibição do dispositivo. Os valores de ISQ variam de 1 a 100 , e quanto maior o ISQ, maior a estabilidade do implante ${ }^{24}$.

Sabe-se que o ISQ>70 corresponde alta estabilidade, entre 60 e 69 média estabilidade e ISQ $<60$ corresponde a baixa estabilidade de acordo com mais de 700 referências, sendo uma ferramenta que substituiu progressivamente outros métodos na pesquisa na Implantodontia ${ }^{23}$. Ao contrário das medidas do torque de inserção, não fornece apenas informações clinicamente relevantes sobre a estabilidade do implante na colocação inicial, mas também pode ser usado para avaliar a estabilidade a qualquer momento posterior do experimento ${ }^{32}$. 
Foram realizados cinco procedimentos cirúrgicos sendo que grupo controle, para acessar a região, foi necessário um novo procedimento cirúrgico, em que o animal passaria pelos mesmos procedimentos anestésicos, a administração de drogas, bem como um novo dano no local da cirurgia. Para realizar as medições, foi realizada uma incisão no ângulo da mandíbula após a palpação do local desejado, a remoção dos cicatrizadores dos implantes, o acoplamento do SmartPeg e finalmente, a mensuração do ISQ com o dispositivo Osstell ${ }^{\mathrm{TM}}$. Devido à complexidade dessa nova etapa cirúrgica, tanto no âmbito da equipe de pesquisa, dos recursos e também os danos ao animal, foi planejado uma maneira de um material que facilitasse essa investigação.

No grupo teste, após a confecção e instalação dos componentes, não foram observadas intercorrências no pós-operatório. A medição do ISQ de implantes dentários instalados na mandíbula de ovelhas foi realizada de tal forma que possibilitasse a avaliação pós-cirúrgica imediata e após 4 semanas sem a necessidade de uma nova cirurgia de reabertura, e futuramente, novas análises em mais períodos, facilitando o manuseio animal sem a necessidade de exposição cirúrgica num segundo estágio.

As mandíbulas de ovelhas foram usadas para determinar mudanças longitudinais na freqüência de ressonância e medidas imediatamente após a instalação dos implantes e após 4 semanas, e observando que os valores de freqüência de ressonância aumentaram ao longo desse tempo no grupo controle, o que não foi obervado no grupo.

São demonstradas outras investigações com implantes na mandíbula de ovelhas $^{13,17,18,33}$ seja no ângulo da mandíbula ou na sua borda inferior, demonstrando efetividade na compreensão dos processos de cicatrização óssea e testes biomecânicos sobre os implantes dentários. Estudos semelhantes também são demonstrados utilizando a crista ilíaca para ancoragem de implantes ${ }^{34}$.

Alguns estudos mostraram que os valores de ISQ aumentam com o tempo após a colocação do implante atingindo um pico entre 6 e 8 semanas. Isso pode estar relacionado ao aumento da rigidez de um implante nos tecidos circundantes que ocorre durante a fase de cicatrização ${ }^{35}$. Em outro estudo, a estabilidade dos 
implantes colocados na tíbia de coelhos foi medida semanalmente durante um período de cicatrização de 15 semanas, demonstrando que os valores de ISQ aumentaram significativamente durante a fase de cicatrização antes de atingir um patamar entre 6 e 8 semanas $^{22}$. Já em outra investigação todos os implantes apresentaram níveis favoráveis de RFA que foram encontrados na terceira semana ${ }^{36}$.

Em contrapartida, outros estudos não demostraram diferenças significativas como um estudo ${ }^{17}$, em que foram instalados implantes no ângulo da mandíbula e feitos testes RFA em vários períodos, sendo que um deles após 4 semanas, mas não apresentaram diferença estatística nos períodos observados e num outro estudo, que não houve diferença estatisticamente significante nos valores médios de RFA medidos após 1 mês de cicatrização².

Alguns resultados diversos mostraram diminuição do valor de RFA durante as primeiras 2 semanas após a colocação do implante, e após esse período um aumento na estabilidade. Esta alteração pode estar relacionada à cicatrização óssea precoce, como mudança biológica e reabsorção óssea alveolar marginal. $O$ remodelamento ósseo reduz o contato primário do osso e no estágio inicial após a colocação do implante, a formação de calo ósseo e o aumento do osso lamelar no osso cortical causam grandes alterações na densidade óssea. Assim, no processo de cicatrização, o contato ósseo primário diminui e o contato ósseo secundário aumenta $36,37,38,39,40$.

Com base nas considerações acima, sugere-se que métodos quantitativos de avaliação da estabilidade do implante em humanos sejam complementares de outros métodos de análise. No caso da utilização dos implantes para reabilitações de pacientes, os exames clínicos e radiográficos também devem ser realizados, procurando características como falta de mobilidade, ausência de dor, ausência de infecção recorrente e ausência de radiolucência contínua ao redor do implante após 3,6 e 12 meses de carregamento, que são considerados critérios que definem o sucesso de um implante ${ }^{10,41,42}$.

Além disso, outros estudos devem distinguir diferentes situações clínicas, tais como carregamento imediato / tardio e locais de implantação pós-extração / 
cicatrizados, já que esse método pode ser usado no momento da inserção do implante, durante o período de cicatrização e quando a prótese dentária está em função ${ }^{42}$.

Sobre a diferença de valores de ISQ encontrados entre os grupos, um estudo verificou a relação entre os valores ISQ entre diferentes pilares protéticos e com a plataforma do implante. Nesse estudo em questão, os transdutores SmartPegs foram fixos aos componentes ou à plataforma do implante e todos os valores nos pilares eram inferiores aos da plataforma do implante, para os mesmos implantes. Os resultados deste estudo sugerem que os valores ISQ obtidos no pilar não podem ser comparados com os valores da plataforma. Esse fenômeno foi explicado pois os transdutores (SmartPegs) imprimem uma força lateral no componente protético quando a medição é realizada, sendo o deslocamento maior, pois aumenta o braço da alavanca em relação à medida feita diretamente na plataforma. Assim, quando a RFA é medida sobre um componente, um valor mais baixo é esperado para o mesmo implante, em comparação com o valor medido diretamente na sua plataforma ${ }^{43}$. Esses mesmos autores sugerem que estudos adicionais devem ser realizados com diferentes diâmetros e conexão do implante, e diferentes componentes para confirmar os achados presentes.

Portanto, a altura do componente sobre a plataforma do implante teve um impacto significativo nas medidas de RFA, quanto maior a altura do pilar, menor o valor do quociente de estabilidade do implante. Esses resultados estão de acordo com os resultados obtidos, em que os valores de RFA foram diferentes no momento da instalação dos implantes e após 4 semanas, quando comparamos ao acoplamento do Smartpeg diretamente na plataforma do implante e ao componente personalizado.

Se o implante não estiver suficientemente estabilizado no momento da colocação, uma cápsula de tecido fibroso pode formar em vez de osso, o que pode levar à falha de osseointegração e posterior falha no implante ${ }^{25}$. O que pode ser explicado no caso das falhas de implantes que obtivemos nesse estudo, sendo que de 32 implantes instalados no total, 5 deles apresentaram falhas após 4 semanas. No entanto, trata-se de um estudo experimental realizado em um modelo animal, 
que envolve uma série de limitações, como a qualidade do osso em uma área diferente da cavidade oral e com um número de amostras reduzido.

Uma outra investigação demonstrou a incorporação de um implante em um bloco de alumínio com alturas diferentes e a obtenção do valor RFA pareceu mudar de acordo com essas alturas, sugerindo uma forte correlação $(r=0,94, p<0,01)$ entre a freqüência observada e a altura da fixação do implante exposta ${ }^{21}$.

De acordo com a literatura, pesquisas demonstram que o sistema RFA contribuído por Osstell ${ }^{\mathrm{TM}}$ contribui com medidas RFA altamente confiáveis em implantes dentários ${ }^{44}$.

Em outro estudo, desenvolveu-se um SmartPeg customizado para realizar medidas de RFA em mini-implantes, demonstrando que desenvolvimento de métodos adicionais são efetivos para a busca de uma melhor compressão dos processos biológicos ${ }^{45}$.

De acordo com os resultados e com a literatura, o uso de componentes personalizados não demonstrou total efetividade quando comparados com o padrão original de medição, ou seja, quando o acoplamento do transdutor SmartPeg ocorre diretamente na plataforma do implante.

Uma compreensão mais profunda da estabilidade do implante durante o período de cicatrização ajuda os clínicos a determinar os protocolos de carga apropriados, diminuindo assim o risco de falha causada por uma fraca estabilidade 
4 CONSIDERAÇÕES FINAIS 



\section{CONSIDERAÇÕES FINAIS}

Após os resultados encontrados, podemos chegar as seguintes conclusões:

A avaliação do componente apresentou resultados satisfatórios no quesito do bem-estar animal e economia de recursos, mas os resultados demonstram que apesar de seus benefícios para a pesquisa, não demonstrou equivalência nos resultados nas medições de ISQ quando comparados ao método convencional.

O uso de ovelhas apresentou uma boa alternativa frente as dificuldades de estudos in vivo, demonstrando biocompatibilidade, disponibilidade e fácil manipulação pela equipe do estudo.

Novos dispositivos e materiais devem ser desenvolvidos com a finalidade de simplificar estudos científicos, já que possuem uma elevada complexidade, principalmente relacionadas com o entendimento de processos biológicos como as que são realizadas em animais.

Estudos in vivo são necessários para entender a situação clínica real em que muitos fatores biológicos influenciam a estabilidade primária dos implantes dentários. 



\section{REFERÊNCIAS}





\section{REFERÊNCIAS}

1. Wancket LM. Animal Models for Evaluation of Bone Implants and Devices. Veterinary Pathology Vol 52, Issue 5, pp. 842 - 850 First published date: July10-2015

2. Duncan W, Lee M, Bae T, Lee S, Gay J, Loch C. Anodisation Increases Integration of Unloaded Titanium Implants in Sheep Mandible. BioMed Research International Vol. 2015.

3. Branemark R, Ohrnell LO, Nilsson P, Thomsen P. Biomechanical characterization of osseointegration during healing: an experimental in vivo study in the rat. Biomaterials. 1997;18(14):969-78. doi: 10.1016/S01429612(97)00018-5.

4. Mehmet Emre Yurttutan, Recep Kestane, Ahmet Keskin,Ömür Dereci. Biomechanical evaluation of oversized drilling on implant stability - an experimental study in sheep. J Pak Med Assoc Vol. 66, No. 2, February 2016

5. Jung UW, Kim S, Kim YH, Cha JK, Lee IS, Choi SH. Osseointegration of dental implants installed without mechanical engagement: a histometric analysis in dogs. ClinOral Implants Res. 2012; 23:1297-301.

6. Shalabi MM, Gortemaker A, Van't hof MA, Jasen JA, Reugers NHJ. Implant surface roughness and bone healing: a systematic review. J Dent Res 2006;85:496-500.

7. Mesa F, Munõs R, Noguerol B, De Dios Luna J, Galindo P, O'valle F. Multivariate study of factors influencing primary dental implant stability. Clin Oral Implants Res 2008;19:196-200.

8. Buser D, Mericske-Stern R, Bernard JP, et al. Long-term evaluation of nonsubmerged ITI implants. Part 1: 8-year life table analysis of a prospective multicenter study with 2359 implants. Clin Oral Implants Res 1997;8:161-172.

9. Hermann JS, Schoolfield JD, Schenk RK, Buser D, Cochran DL. Influence of the size of the microgap on crestal bone changes around titanium implants: a histometric evaluation of unloaded non-submerged implants in the canine mandible. J Periodontol. 2001;72:1372-1383. 
10. Cochran DL, Buser D, ten Bruggenkate CM, et al. The use of reduced healing times on ITI implants with a sandblasted and acid-etched (SLA) surface: Early results from clinical trials on ITI SLA implants. Clin Oral Implants Res 2002;13:144-153.

11. Sartoretto SC, Alves ATNN, Zarranz L, Jorge MZ, Granjeiro JM, CalasansMaia MD. Hydrophilic surface of Ti6Al4V-ELI alloy improves the early bone apposition of sheep tibia. Clin. Oral Impl. Res. 00, 2016, 1-9 doi: 10.1111/clr.12894

12. Trisi $P$, Berardini M, Falco A, Podaliri Vulpiani M. New Osseodensification Implant Site Preparation Method to Increase Bone Density in Low-Density Bone: In Vivo Evaluation in Sheep. Implant Dentistry. 2016;25(1):24-31. doi:10.1097/ID.0000000000000358.

13. Bowers M, Yoo D, Marin C, Gil L, Shabaka N, Goldstein M, Janal M, Tovar N, Hirata R, Bonfante E, Coelho P. Surface characterization and in vivo evaluation of laser sintered and machined implants followed by resorbable-blasting media process: A study in sheep. Med Oral Patol Oral Cir Bucal. 2016 Mar 1;21 (2):e206-13.

14. Duncan, WJ. Sheep mandibular animal models dental implantology research. A thesis submitted for the degree of Doctor of Philosophy at the University of Otago, Dunedin, New Zealand, Jan. 2005.

15. Yoo D, Marin C, Freitas G, Tovar N, Bonfante E, Teixeira H, Janal M, Coelho P. Surface Characterization and In Vivo Evaluation of Dual Acid-Etched and GritBlasted/Acid-Etched Implants in Sheep. Implant Dentistry. Vol. 24 N. 3, 2015.

16. Ernst S, Stubinguer S, Schupbach P, Sidler M, Klein K., Ferguson S, Rechenberg B. Comparison of two dental implant surface modifications on implants with same macrodesign: an experimental study in the pelvic sheep model. Clin. Oral Impl. Res. 26, 2015, 898-908.

17. Trisi $P$, Todisco $M$, Consolo $U$, Travaglini, D. High versus low implant insertion torque: a histologic, histomorphometric, and biomechanical study in the sheep mandible. Int J Oral Maxillofac Implants. 2011 Jul-Aug;26(4):837-49.

18. Dagher M, Mokbel N, Jabbour G, Naaman N. Resonance Frequency Analysis, Insertion Torque, and Bone to Implant Contact of 4 Implant Surfaces: Comparison and Correlation Study in Sheep. Implant Dentistry. 2014 vol. 23 n. 6. 
19. Xiao J, Li D, Chen Y, Chen N S, Kong, L. Evaluation of Fixation of Expandable Implants in the Mandibles of Ovariectomized Sheep. J Oral Maxillofac Surg 71:682-688, 2013.

20. Atsumi M, Park SH, Wang HL. Methods used to assess implant stability: current status. Int J Oral Maxillofac Implants. 2007;22(5):743-54.

21. Meredith N, Alleyne D, Cawley P. Quantitative determination of the stability of the implant-tissue interface using resonance frequency analysis. Clin Oral Implants Res. 1996;7(3):261-7. doi: 10.1034/j.1600-0501.1996.070308.x.

22. Meredith N, Shagaldi F, Alleyne D, Sennerby L, Cawley P. The application of resonance frequency measurements to study the stability of titanium implants during healing in the rabbit tibia. Clin Oral Implants Res. 1997;8(3):234-43. doi: 10.1034/j.1600-0501.1997.080310.x

23. Bischof M, Nedir R, Szmukler-Moncler S, Bernard JP, Samson J. Implant stability measurement of delayed and immediately loaded implants during healing. Clin Oral Implants Res. 2004;15:529-539.

24. Alsaadi G, Quirynen M, Michiels K, Jacobs R, van Steenberghe D. A biomechanical assessment of the relation between the oral implant stability at insertion and subjective bone quality assessment. J Clin Periodontol 2007;34:359-366.

25. Santamaría-Arrieta, G, Brizuela-Velasco A, Fernández-González F, ChávarriPrado D, Chento-Valiente Y, Solaberrieta E, Yurrebaso-Asúa, J. (2016). Biomechanical evaluation of oversized drilling technique on primary implant stability measured by insertion torque and resonance frequency analysis. Journal of Clinical and Experimental Dentistry, 8(3), e307-e311. http://doi.org/10.4317/jced.52873

26. Scarano A, Lorusso F, Ravera L, Mortellaro C, Piattelli A. Bone Regeneration in Iliac Crestal Defects: An Experimental Study on Sheep. BioMed Research International. 2016;2016:4086870. doi:10.1155/2016/4086870.

27. Alayan J, Vaquette C, Saifzadeh S, Hutmacher D, Ivanovski S. Comparison of early osseointegration of SLA and SLActive implants in maxillary sinus augmentation: a pilot study. Clin. Oral Impl. Res. 00, 2016, 1-9 doi: $10.1111 /$ clr.12988

28. Turner AS. Experiences with sheep as an animal model for shoulder surgery Strengths and shortcomings. J Shoulder Elbow Surg. 2007;16(5):158S-163S. 
29. Sartoretto SC, Uzeda MJ, Miguel FB, Nascimento JR, Ascoli F, Calasans-maia MD. Sheep as an experimental model for biomaterial implant evaluation. Acta Ortopedica Brasileira. 2016;24(5):262-266. doi:10.1590/1413785220162405161949.

30. NC3Rs Reporting Guidelines Working Group (2010) Animal research: reporting in vivo experiments: the ARRIVE guidelines. The Journal of Physiology 588: 2519-2521.

31. Trisi P, Berardini M, Falco A, Podaliri Vulpiani M. Effect of Implant Thread Geometry on Secondary Stability, Bone Density, and Bone-to-ImplantContact: A Biomechanical and Histological Analysis. Implant Dent. 2015 Aug;24(4):38491. doi: 10.1097/ID.0000000000000269

32. Trisi P, Berardini M, Falco A, Sandrini E, Vulpiani MP. A New Highly Hydrophilic Electrochemical Implant Titanium Surface: A Histological and Biomechanical In Vivo Study. Implant Dent. 2017 Jun;26(3):429-437. doi: 10.1097/ID.0000000000000605.

33. Huang HM, Chiu CL, Lin CT, Lin LH, Lee SY. Early detection of implant healing process using resonance frequency analysis. Clin Oral Implants Res. 2003;14:437-443).

34. Abdel-Haq, J., Karabuda, CZ, Arısan, V., Mutlu, Z. e Kürkçü, M. Osseointegração e estabilidade de um implante de ácido acidificado: um estudo piloto experimental em ovelhas. Clinical Oral Implants Research, 22: 265-274, 2011 doi: 10.1111 / j.1600-0501.2010.01990.x

35. Satwalekar, P., Nalla, S., Reddy, R., \& Chowdary, S. G. (2015). Clinical evaluation of osseointegration using resonance frequency analysis. The Journal of the Indian Prosthodontic Society, 15(3), 192-199. http://doi.org/10.4103/0972-4052.165171

36. Perrotti V, Piattelli A, lezzi G. Mineralized bone-implant contact and implant stability quotient in 16 human implants retrieved after early healing periods: $A$ histologic and histomorphometric evaluation. Int J Oral Maxillofac Implants. 2010;25:45-8.)

37. Sim, CPC, Lang NP. Factors influencing resonance frequency analysis assessed by Osstell ${ }^{\mathrm{TM}}$ mentorduring implant tissue integration: I. Instrument positioning, bone structure, implant length. Clinical Oral Implants Research 2010, 21: 598-604. doi:10.1111/j.1600-0501.2009.01878.x). 
38. Salata LA., Burgos PM, Rasmusson L, et al. Osseointegration of oxidized and turned implants in circumferential bone defects with and without adjunctive therapies: an experimental study on BMP-2 and autogenous bone graft in the dog mandible. International Journal of Oral and Maxillofacial Surgery. 2007;36(1):62-71. doi: 10.1016/j.ijom.2006.07.009.

39. Sul YT, Jönsson J, Yoon GS, Johansson C. Resonance frequency measurements in vivo and related surface properties of magnesiumincorporated, micropatterned and magnesium-incorporated TiUnite, Osseotite, SLA and TiOblast implants. Clinical Oral Implants Research. 2009;20(10):1146-1155. doi: 10.1111/j.1600-0501.2009.01767.x.

40.Zix J, Hug S, Kessler-Liechti G, Mericske-Stern R. Measurement of dental implant stability by resonance frequency analysis and damping capacity assessment: Comparison of both techniques in a clinical trial. Int $J$ Oral Maxillofac Implants 2008;23:525-530.

41. Andreotti AM, Goiato MC, Nobrega AS, et al. Relationship between implant stability measurements obtained by two different devices: a systematic review. J Periodontol. 2017;88(3):281-288.

42. Lages FS, Douglas-de Oliveira DW, Costa FO. Relationship between implant stability measurements obtained by insertion torque and resonance frequency analysis: A systematic review. Clin Implant Dent Relat Res. 2018;20:26-33. https://doi.org/10.1111/cid.12565).

43. Lages, FS, Willya Douglas-de-Oliveira D, Ibelli, GS, Assaf F, Queiroz TP, Costa FO. (2017). Relationship between implant stability on the abutment and platform level by means of resonance frequency analysis: A cross-sectional study. PLoS ONE, 12(7), e0181873. http://doi.org/10.1371/journal.pone.0181873)

44. Herrero-Climent, M, Albertini M, Rios-Santos JV, Lázaro-Calvo P, FernándezPalacín A, Bullon P. (2012). Resonance frequency analysis-reliability in third generation instruments: Osstell mentor $\circledast$. Medicina Oral, Patología Oral Y Cirugía Bucal, 17(5), e801-e806. http://doi.org/10.4317/medoral.17861.

45. Dhaliwal JS, Albuquerque RF, Fakhry A, Kaur S, Feine JS. Customized SmartPeg for measurement of resonance frequency of mini dental implants. International Journal of Implant Dentistry. 2017;3:4. doi:10.1186/s40729-017-0066-6. 
46. Barewal RM, Oates TW, Meredith N, Cochran DL. Resonance frequency measurement of implant stability in vivo on implants with a sandblasted and acid-etched surface. Int J Oral Maxillofac Implants. 2003;18(5):641-51

47. Glauser R, Sennerby L, Meredith N, Ree A, Lundgren A, Gottlow J, et al. Resonance frequency analysis of implants subjected to immediate or early functional occlusal loading. Successful vs. failing implants. Clin Oral Implants Res. 2004;15(4):428-34. doi: 10.1111/j.1600-0501.2004.01036.x

48. Binon PP. Implants and components: Entering the new millennium. Int J Oral Maxillofac Implants. 2000;15:76-94.

49. Branemark PI, Hansson BO, Adel R. Osseointegrated implants in the treatment of the edentulous jaw. Experience from a 10-year period. Scand J Plast Reconstr. 1977;16:1-132. 1

50. Branemark PI, Adell R, Breune U, Hansson BO, Lindstrom J, Ohlsson A. A. Intra-osseous anchorage of dental prostheses. Scand J Palst Reconstr Surg, 1969, v.3, p.81-100.

51. Cooper L, Felton DA, Kugelberg CF. A multicenter 12-month evaluation of single-tooth implants restored 3 weeks after 1-stage surgery. Int J Oral Maxillofac Implants. 2001;16:182-192.

52. Novaes AB, Souza SL, De Oliveira PT, Souza AM. Histomorphometric analysis of the boné-implant contact obtained with 4 different implant surface treatments placed side by side in the dog mandible. Int $\mathrm{J}$ Oral Maxillofac Implants. 2002;17:377-383.

53. Novaes JR AB, De Oliveira RR, Muglia VA, Papalexiou V, Taba M. The effects of interimplant distances on papilla formation and crestal resorption in implants with a morse cone connection and a platform switch: a histomorphometric study in dogs. J Periodontol. Nov 2006a;77(11):1839-49.

54. Piattelli A, Vrespa G, Petrone G, lezzi G, Annibali S. Role of the microgap between implant and abutment: a retrospective histologic evaluation in monkeys. J Periodontol. 2003; 74:346-352.

55. Schwarz MLR, Kowarsch M, Rose S, Becker K, Lenz T, Jani L. Effect of surface roughness, porosity, and a resorbable calcium phosphate coating on osseointegration of titanium in a minipig model. Journal of Biomedical Materials Research Part A. 2009, v. 89A(3), p. 667-678. 
ANEXOS 



\section{NEXO A - Aprovação do Comitê de ética em animais de Pirassununga}

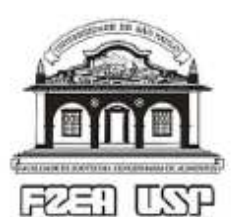

UNIVERSIDADE DE SĀO PAULO

Faculdade de Zootecria e Engenharia de Alimentos Comite de t́tica em Pesquisa da FZEA

\section{CERTIFICADO}

Certificamos que a proposta intitulada "Estudo comparativo de dois diferentes tipos de tratamento de superficies em implantes dentários no modelo animal $\square$ ovelha[ ", protocolada sob o CEUA $n^{2} 5481280616$, sob a responsabilidade de Adriano Bonfim Carregaro - que envolve a produçăo, manutenção e/ou utilização de animais pertencentes ao filo Chordata, subfilo Vertebrata (exceto o homem), para fins de pesquisa científica ou ensino - está de acordo com os preceitos da Lei 11.794 de 8 de outubro de 2008, com o Decreto 6.899 de 15 de julho de 2009, bem como com as normas editadas pelo Conselho Nacional de Controle da Experimentação Animal (CONCEA), e foi aprovada pela Comissão de Ética no Uso de Animais da Faculdade de Zootecnia e Engenharia de Alimentos da Universidade de São Paulo - FZEA/USP (CEUA/FZEA) na reuniăo de 20/12/2016.

We certify that the proposal "Comparison of two different types of surface treatment in dental implants in the animal model "sheep" ", utilizing 5 Ovines (males and females), protocol number CEUA 5481280616, under the responsibility of Adriano Bonfim Carregaro - which involves the production, maintenance and/or use of animals belonging to the phylum Chordata, subphylum Vertebrata (except human beings), for scientific research purposes or teaching - is in accordance with Law 11.794 of October 8 , 2008, Decree 6899 of July 15, 2009, as well as with the rules issued by the National Council for Control of Animal Experimentation (CONCEA), and was approved by the Ethic Committee on Animal Use of the School of Animal Science and Food Engineering - (São Paulo University) (CEUA/FZEA) in the meeting of 12/20/2016.

Finalidade da Proposta: Pesquisa (Acadêmica)

Vigência da Proposta: de 07/2016 a 07/2017_Área: Medicina Veterinária

Origem: $\quad$ Animais de proprietários

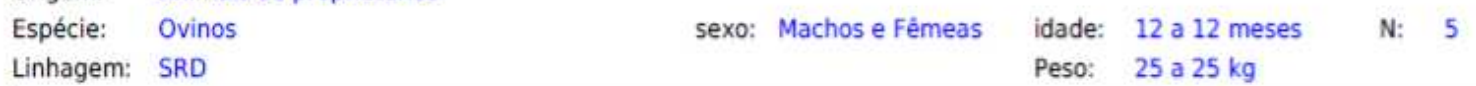

Resumo: A união entre osso e implante não tem suas características bem estabelecidas na literatura. A literatura afirma que o titânio comercialmente puro (TiCp ou grau 4) é um material que possui excelentes propriedades de biocompatibilidade óssea devido a sua composiçăo química, favorecendo assim o sucesso da osseointegraçăo. Um fator que vem sendo alvo de muita discussăo é o tipo de tratamento da superfície dos implantes, que aceleram o tempo de osseointegraçāo. Essas superficies tratadas também possuem propriedades acopladas que podem Haverá abate dos animais? Sim Segue orientações do COBEA? Sim Espécie: Ovinos Gênero: Machos e Fêmeas Peso: 25 idade: 12 meses Linhagem: SRD Instalação: Baia Tipo de cama: Maravalha $\mathrm{N}$ por área: 1/m2 N: 5 Biotério: Não aplicável Procedência: Os animais serão adquiridos de proprietários Manutenção: Os animais serão mantidos nas instalaçōes Condiçōes de alojamento: lluminaçăo: 12 horas / iluminaçăo natural Alimentação: 2 vezes ao dia (raçāo e feno) Fonte de água: ad libitum (filtrada) Exaustâo de ar: natural melhorar o seu desempenho, como a molhabilidade. 0 presente estudo propōe a utilizaçăo dos Implantes Cone-Morse com o intuito de comparar o grau de osseointegraçăo através do destorque e análise de frequência de ressonância de implantes, com tratamento de superfície microtexturizada e propriedade de superficie aquosa com a análise histomorfométrica. Para tal estudo, serão utilizadas 5 ovelhas e instalados no total, 40 implantes que serão instalados no ângulo da mandíbula com torque de $45 \mathrm{Ncm}$ e passarăo por avaliação em 5 períodos diferentes: 1, 2, 3, 4 e 6 semanas. Após cada período estipulado, os ovinos passarão pelos testes, serão eutanasiados e suas mandibulas seccionadas. fixadas e incluídas para a avaliação histomorfométrica. Os resultados serâo avaliados através da análise estatistica de ANOVA e Teste $[t]$ variáveis independentes. Será adotado nivel de significância de $5 \%(p<0,05)$.

Local do experimento: Os procedimentos de cirurgia e manutenção serão realizados na UDCH da FZEA-USP

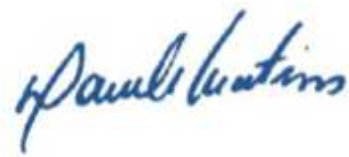

Profa. Dra. Daniele dos Santos Martins Coordenadora da Comissão de Ética no Uso de Animais

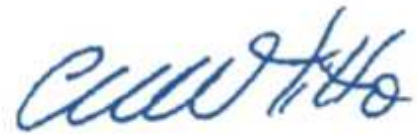

Profa. Dra. Cristiane Gonçalves Titto Vice-Coordenadora da Comissăo de Ética no Uso de Animais 


\section{ANEXO B - Diretrizes para The Journal of Prosthetic Dentistry:}

\section{Submission Guidelines}

Thank you for your interest in writing an article for The Journal of Prosthetic Dentistry. In publishing, as in dentistry, precise procedures are essential. Your attention to and compliance with the following policies will help ensure the timely processing of your submission.

\section{Length of Manuscripts}

Manuscript length depends on manuscript type. In general, research and clinical science articles should not exceed 10 to 12 double-spaced, typed pages (excluding references, legends, and tables). Clinical Reports and Technique articles should not exceed 4 to 5 pages, and Tips articles should not exceed 1 to 2 pages. The length of systematic reviews varies.

\section{Number of Authors}

The number of authors is limited to 4 ; the inclusion of more than 4 must be justified in the letter of submission. (Each author's contribution must be listed.) Otherwise, contributing authors in excess of 4 will be listed in the Acknowledgments. There can only be one corresponding author.

\section{General Formatting}

All submissions must be submitted via the EES system in Microsoft Word with an $8.5 \times 11$ inch page size. The following specifications should also be followed:

Times Roman, 12 ptDouble-spacedLeft-justifiedNo space between paragraphs1-inch margins on all sidesHalf-inch paragraph indentsHeaders/Footers should be clear of page numbers or other informationHeadings are upper case bold, and subheads are upper/lower case bold. No italics are used.References should not be automatically numbered. Endnote or other reference-generating programs should be turned off.Set the Language feature in MS Word to English (US). Also change the language to English (US) in the style named Balloon Text.

Ethics in publishing

Please see our information pages on Ethics in publishing and Ethical guidelines for journal publication.

\section{Declaration of interest}

All authors must disclose any financial and personal relationships with other people or organizations that could inappropriately influence (bias) their work. Examples of potential conflicts of interest include employment, consultancies, stock ownership, honoraria, paid expert testimony, patent applications/ registrations, and grants or other funding. If there are no conflicts of interest then please state this: 'Conflicts of interest: none'. More information.

\section{Submission declaration}

Submission of an article implies that the work described has not been published previously (except in the form of an abstract or as part of a published lecture or academic thesis or as an electronic preprint, see 'Multiple, redundant or concurrent publication' section of our ethics policy for more information), that it is not under consideration for publication elsewhere, that its publication is approved by all authors and tacitly or explicitly by the responsible authorities where the work was carried out, and that, if accepted, it will not be published elsewhere including electronically in the same form, in English or in any other language, without the written consent of the copyright-holder.

\section{Changes to authorship}

Authors are expected to consider carefully the list and order of authors before submitting their manuscript and provide the definitive list of authors at the time of the original submission. Any addition, deletion or rearrangement of author names in the authorship list should be made only before the manuscript has been accepted and only if approved by the journal Editor. To request such a change, the Editor must receive the following from the corresponding author: (a) the reason for the change in author list and (b) written confirmation (e-mail, letter) from all authors that they agree with the addition, removal or rearrangement. In the case of addition or removal of authors, this includes confirmation from the author being added or removed.

Only in exceptional circumstances will the Editor consider the addition, deletion or rearrangement of authors after the manuscript has been accepted. While the Editor considers the request, publication of the manuscript will be suspended. If the manuscript has already been published in an online issue, any requests approved by the Editor will result in a corrigendum. 


\section{Copyright}

Upon acceptance of an article, authors will be asked to complete a 'Journal Publishing Agreement' (see more information on this). An e-mail will be sent to the corresponding author confirming receipt of the manuscript together with a 'Journal Publishing Agreement' form or a link to the online version of this agreement.

Subscribers may reproduce tables of contents or prepare lists of articles including abstracts for internal circulation within their institutions. Permission of the Publisher is required for resale or distribution outside the institution and for all other derivative works, including compilations and translations. If excerpts from other copyrighted works are included, the author(s) must obtain written permission from the copyright owners and credit the source(s) in the article. Elsevier has preprinted forms for use by authors in these cases.

For open access articles: Upon acceptance of an article, authors will be asked to complete an 'Exclusive License Agreement' (more information). Permitted third party reuse of open access articles is determined by the author's choice of user license.

\section{Author rights}

As an author you (or your employer or institution) have certain rights to reuse your work. More information.

Elsevier supports responsible sharing

Find out how you can share your research published in Elsevier journals.

\section{Role of the funding source}

You are requested to identify who provided financial support for the conduct of the research and/or preparation of the article and to briefly describe the role of the sponsor(s), if any, in study design; in the collection, analysis and interpretation of data; in the writing of the report; and in the decision to submit the article for publication. If the funding source(s) had no such involvement then this should be stated.

Funding body agreements and policies

Elsevier has established a number of agreements with funding bodies which allow authors to comply with their funder's open access policies. Some funding bodies will reimburse the author for the Open Access Publication Fee. Details of existing agreements are available online.

Creative Commons Attribution (CC BY)

Lets others distribute and copy the article, create extracts, abstracts, and other revised versions, adaptations or derivative works of or from an article (such as a translation), include in a collective work (such as an anthology), text or data mine the article, even for commercial purposes, as long as they credit the author(s), do not represent the author as endorsing their adaptation of the article, and do not modify the article in such a way as to damage the author's honor or reputation.

Creative Commons Attribution-NonCommercial-NoDerivs (CC BY-NC-ND)

For non-commercial purposes, lets others distribute and copy the article, and to include in a collective work (such as an anthology), as long as they credit the author(s) and provided they do not alter or modify the article.

The open access fee for this journal is USD 2500, excluding taxes. Learn more about Elsevier's pricing policy: https://www.elsevier.com/openaccesspricing.

Language (usage and editing services)

Please write your text in good American English. Authors who feel their English language manuscript may require editing to eliminate possible grammatical or spelling errors and to conform to correct scientific English may wish to use the English Language Editing service available from Elsevier's WebShop http://webshop.elsevier.com/languageediting/ or visit our customer support site http://support.elsevier.com for more information.

\section{Informed consent and patient details}

Studies on patients or volunteers require ethics committee approval and informed consent, which should be documented in the paper. Appropriate consents, permissions and releases must be obtained where an author wishes to include case details or other personal information or images of patients and any other individuals in an Elsevier publication. Written consents must be retained by the author and copies of the consents or evidence that such consents have been obtained must be provided to 
Elsevier on request. For more information, please review the Elsevier Policy on the Use of Images or Personal Information of Patients or other Individuals. Unless you have written permission from the patient (or, where applicable, the next of kin), the personal details of any patient included in any part of the article and in any supplementary materials (including all illustrations and videos) must be removed before submission.

\section{Submission}

Our online submission system guides you stepwise through the process of entering your article details and uploading your files. The system converts your article files to a single PDF file used in the peer-review process. Editable files (e.g., Word, LaTeX) are required to typeset your article for final publication. All correspondence, including notification of the Editor's decision and requests for revision, is sent by e-mail.

Submit your article

Please submit your article via http://www.ees.elsevier.com/jpd/.

\section{PREPARATION}

Use of word processing software

It is important that the file be saved in the native format of the MS Word program. The text should be in single-column format. Keep the layout of the text as simple as possible. Most formatting codes will be removed and replaced on processing the article. In particular, do not use the word processor's options to justify text or to hyphenate words. However, do use bold face, italics, subscripts, superscripts etc. When preparing tables, if you are using a table grid, use only one grid for each individual table and not a grid for each row. If no grid is used, use tabs, not spaces, to align columns. The electronic text should be prepared in a way very similar to that of conventional manuscripts (see also the Guide to Publishing with Elsevier: http://www.elsevier.com/guidepublication). Note that source files of figures, tables and text graphics will be required whether or not you embed your figures in the text. See also the section on Electronic artwork.

To avoid unnecessary errors you are strongly advised to use the 'spell-check' and 'grammar-check' functions of your word processor.

Embedded math equations
embedded math equations then please read this related support information
(http://support.elsevier.com/app/answers/detail/a_id/302/).

\section{Essential title page information}

Title. Concise and informative. Titles are often used in information-retrieval systems. Avoid abbreviations and formulae. Trade names should not be used in the title.Author names and affiliations. Author's names should be complete first and last names. Where the family name may be ambiguous (e.g., a double name), please indicate this clearly. Present the authors' current title and affiliation, including the city and state/country of that affiliation. If it is private practice, indicate the city and state/country of the practice. Indicate all affiliations with a lower-case superscript letter immediately after the author's name and in front of the appropriate affiliation.Corresponding author. Clearly indicate who will handle correspondence at all stages of refereeing and publication, also postpublication. Ensure that phone numbers (with country and area code) are provided in addition to the e-mail address and the complete postal address. Contact details must be kept up to date by the corresponding author.

Title page format

Title: Capitalize only the first letter of the first word. Do not use any special formatting. Abbreviations or trade names should not be used. Trade names should not be used in the title.Authors: Directly under the title, type the names and academic degrees of the authors. Under the authors names, provide the title, department and institutional names, city/state and country (unless in the U.S.) of each author. If necessary, provide the English translation of the institution. If the author is in private practice, indicate where with city/state/country. Link names and affiliations with a superscript letter $(a, b, c, d)$. Presentation/support information and titles: If research was presented before an organized group, indicate name of the organization and location and date of the meeting. If work was supported by a grant or any other kind of funding, supply the name of the supporting organization and the grant 
number. Corresponding author: List the mailing address, business telephone, and e-mail address of the author who will receive correspondence. Acknowledgments: Indicate special thanks to persons or organizations involved with the manuscript. See Sample Title page.

\section{Formatting of funding sources}

List funding sources in this standard way to facilitate compliance to funder's requirements:

Funding: This work was supported by the National Institutes of Health [grant numbers xxxx, yyyy]; the Bill \& Melinda Gates Foundation, Seattle, WA [grant number zzzz]; and the United States Institutes of Peace [grant number aaaa].

It is not necessary to include detailed descriptions on the program or type of grants and awards. When funding is from a block grant or other resources available to a university, college, or other research institution, submit the name of the institute or organization that provided the funding.

If no funding has been provided for the research, please include the following sentence:

This research did not receive any specific grant from funding agencies in the public, commercial, or not-for-profit sectors.

Units

Follow internationally accepted rules and conventions: use the international system of units (SI). If other units are mentioned, please give their equivalent in SI.

Math formulae

Please submit math equations as editable text and not as images. Present simple formulae in line with normal text where possible and use the solidus $(/)$ instead of a horizontal line for small fractional terms, e.g., X/Y. In principle, variables are to be presented in italics. Powers of e are often more conveniently denoted by exp. Number consecutively any equations that have to be displayed separately from the text (if referred to explicitly in the text).

Embedded math equations

If you are submitting an article prepared with Microsoft Word containing embedded math equations then please read this (related support information).

\section{Artwork \\ Figure Submission}

JPD takes pride in publishing only the highest quality figures in its journal. All incoming figures must pass a thorough examination in Photoshop before the review process can begin. With more than 1,000 manuscripts submitted yearly, the manuscripts with few to no submission errors move through the system quickly. Figures that do not meet the guidelines will be sent back to the author for correction and moved to the bottom of the queue, creating a delay in the publishing process.

File Format

All figures should be submitted as TIF files or JPEG files only.

\section{Image File Specifications}

\section{Figure dimensions must be $\mathbf{5 . 7 5} \times \mathbf{3 . 8 5}$ inches.}

Figures should be size-matched (the same physical size) unless the image type prohibits size matching to other figures within the manuscript, as in the case of panoramic or periapical radiographs, SEM images, or graphs and screen shots. Do not "label" the faces of the figures with letters or numbers to indicate the order in which the figures should appear; such labels will be inserted during the publication process. Do not add wide borders to increase size.

\section{Resolution}

The figures should be of professional quality and high resolution. The following are resolution requirements:

Color and black-and-white photographs should be created and saved at 300 dots per inch (dpi).

Note: A $5.75 \times 3.85$-inch image at a resolution of $300 \mathrm{dpi}$ will be approximately 6 megabytes. A figure of less than $300 \mathrm{dpi}$ must not be increased artificially to $300 \mathrm{dpi}$; the resulting quality and resolution will be poor. 
Line art or combination artwork (an illustration containing both line art and photograph) should be created and saved at a minimum of 600dpi.Clarity, contrast, and quality should be uniform among the parts of a multipart figure and among all of the figures within a manuscript.A uniform background of nontextured, medium blue should be provided for color figures when possible.

\section{Text within Images}

If text is to appear within the figure, labeled and unlabeled versions of the figures must be provided. Text appearing within the labeled versions of the figures should be in Arial font and a minimum of $10 \mathrm{pt}$. The text should be sized for readability if the figure is reduced for production in the Journal. Lettering should be in proportion to the drawing, graph, or photograph. A consistent font size should be used throughout each figure, and for all figures, Please note: Titles and captions should not appear within the figure file, but should be provided in the manuscript text (see Figure Legends).

If a key to an illustration requires artwork (screen lines, dots, unusual symbols), the key should be incorporated into the drawing instead of included in the typed legend. All symbols should be done professionally, be visible against the background, and be of legible proportion should the illustration be reduced for publication.

All microscopic photographs must have a measurement bar and unit of measurement on the image.

\section{Color Figures}

Generally, a maximum of 8 figures will be accepted for clinical report and dental technique articles, and 2 figures will be accepted for tips from our reader articles. However, the Editor may approve the publication of additional figures if they contribute significantly to the manuscript.

Clinical figures should be color balanced. Color images should be in CMYK (Cyan/Magenta/Yellow/ Black) color format as opposed to RGB (Red/Green/Blue) color format.

\section{Graphs/Screen Captures}

Graphs should be numbered as figures, and the fill for bar graphs should be distinctive and solid; no shading or patterns. Thick, solid lines should be used and bold, solid lettering. Arial font is preferred. Place lettering on white background is preferred to reverse type (white lettering on a dark background). Line drawing should be a minimum of $600 \mathrm{dpi}$. Screen Captures should be a minimum of $300 \mathrm{dpi}$ and as close to 5.75 and 3.85 as possible.

\section{Composites}

Composites are multiple images within one Figure file and, as a rule, are not accepted. They will be sent back to the author to replace them with each image sent separately as, Fig. 1A, Fig. 1B, Fig. 1C, etc. Each figure part must meet JPD Guidelines. (Some composite figures are more effective when submitted as one file. These files will be reviewed per case.) Contact the editorial office for more information about specific composites.

\section{Figure Legends}

The figure legends should appear within the text of the manuscript on a separate page after Tables and should appear under the heading FIGURES. Journal style requires that the articles ( $a$, an, and the) are omitted from the figure legends. If an illustration is taken from previously published material, the legend must give full credit to the source (see Permissions).

\section{File Naming}

Each figure file must be numbered according to its position in the text (Figure 1, Figure 2, and so on) with Arabic numerals. The electronic image files must be named so that the figure number and format can be easily identified. For example, a Figure 1 in TIFF format should be named fig. 1.tif. Multipart figures must be clearly identifiable by the file names: Fig. 1A, Fig. 1B, Fig. 1C, Fig. 1-unlabeled, Fig. 1-labeled, etc.

Callouts 
In the article, clearly reference each Figure and Table by including its number in parentheses at the end of the appropriate sentence before closing punctuation. For example: The sutures were removed after 3 weeks (Fig. 4). Or: are illustrated in Table 4.

The Journal reserves the right to standardize the format of graphs and tables.

Authors are obligated to disclose whether illustrations have been modified in any way.

\section{Thumbnails}

Place thumbnails (reduced size versions) of your figures in Figures section below each appropriate legend.

Thumbnails refers to placing a small (compressed file) copy of your figure into the FIGURES section of the manuscript after each appropriate legend. No smaller than $2^{\prime \prime} \times 1.5^{\prime \prime}$ and approximately $72 \mathrm{dpi}$. The goal is to give the editors/reviewers something to review but we want to keep the dimensions and the file size small for easy access. These small images are called thumbnails.

Figures Quick Checklist

All files are saved as TIFFs or JPEGs (only). Figure size: $5.75^{\prime \prime} \times 3.85^{\prime \prime}$ (radiographs, SEMS, and screen captures may vary but they must all be size-matched). Figures are $300 \mathrm{dpi}$; line or combo line/photo illustrations are minimum $600 \mathrm{dpi}$. For text in figures use Ariel font.Label the Figure files according to their sequence in the text.Provide figure legends in the manuscript Figure section.Place thumbnails (small versions of figure files approx. $2^{\prime \prime} \times 1.5^{\prime \prime}$ ) in Figure section below each legend. Submit composite figure parts as separate files.

A detailed guide to electronic artwork is available on our website: You are urged to visit this site; some excerpts from the detailed information about figure preparation are given here. http://www.elsevier.com/artworkinstructions.

Please make sure that artwork files are TIFFs and with the correct resolution. If, together with your accepted article, you submit usable color figures then Elsevier will ensure, at no additional charge, that these figures will appear in color online (e.g., ScienceDirect and other sites) in addition to color reproduction in print. For further information on the preparation of electronic artwork, please see http://www.elsevier.com/artworkinstructions.

\section{Illustration services}

Elsevier's WebShop (http://webshop.elsevier.com/illustrationservices) offers Illustration Services to authors preparing to submit a manuscript but concerned about the quality of the images accompanying their article. Elsevier's expert illustrators can produce scientific, technical, and medicalstyle images, as well as a full range of charts, tables, and graphs. Image 'polishing' is also available, where our illustrators take your image(s) and improve them to a professional standard. Please visit the website to find out more.

\section{Electronic Artwork}

General points

- Make sure you use uniform lettering and sizing.

- Embed the used fonts if the application provides that option.

- Use the font Ariel or Helvetica in your illustrations.

- Number the illustration files according to their sequence in the text.

- Use a logical naming convention for your artwork files.

- Provide figure legends in the Figure section.

- Size the illustrations close to the desired dimensions of the published version.

- Submit each illustration as a separate file.

A detailed guide on electronic artwork is available on our website:

http://www.elsevier.com/artworkinstructions.You are urged to visit this site; some excerpts from the detailed information are given here.

Formats

If your electronic artwork is created in a Microsoft Office application (Word, PowerPoint, Excel) then please supply 'as is' in the native document format.

Regardless of the application used other than Microsoft Office, when your electronic artwork is finalized, please 'Save as' or convert the images to one of the following formats (note the resolution requirements for line drawings, halftones, and line/halftone combinations given below):

TIFF (or JPEG): Color or grayscale photographs (halftones), keep to a minimum of $300 \mathrm{dpi}$.

TIFF (or JPEG): Bitmapped (pure black \& white pixels) line drawings, keep to a minimum of 600 dpi. TIFF (or JPEG): Combinations bitmapped line/half-tone (color or grayscale), keep to a minimum of 600 dpi.

Please do not: 
- Supply files that are optimized for screen use (e.g., GIF, PNG, PICT, WPG); these typically have a low number of pixels and limited set of colors;

- Supply files that are too low in resolution? or smaller than $5.75 \times 3.85$-inch.;

- Submit graphics that are disproportionately large for the content.

\section{Color artwork}

Please make sure that artwork files are in an acceptable format (TIFF or JPEG)and with the correct size and resolution. If, together with your accepted article, you submit usable color figures then Elsevier will ensure, at no additional charge, that these figures will appear in color online (e.g., ScienceDirect and other sites) in addition to color reproduction in print. For further information on the preparation of electronic artwork, please see http://www.elsevier.com/artworkinstructions.

\section{Illustration services}

Elsevier's WebShop offers Illustration Services to authors preparing to submit a manuscript but concerned about the quality of the images accompanying their article. Elsevier's expert illustrators can produce scientific, technical and medical-style images, as well as a full range of charts, tables and graphs. Image 'polishing' is also available, where our illustrators take your image(s) and improve them to a professional standard. Please visit the website to find out more.

\section{Figure captions}

Ensure that each illustration has a caption. Supply captions separately, not attached to the figure. A caption should comprise a brief title (not on the figure itself) and a description of the illustration. Keep text in the illustrations themselves to a minimum but explain all symbols and abbreviations used. See Sample Figures page.

\section{Tables}

Tables should be self-explanatory and should supplement, not duplicate the text. Provide all tables at the end of the manuscript after the reference list and before the Figures. There should be only one table per page. Omit internal horizontal and vertical rules (lines). Omit any shading or color.Do not list tables in parts (Table Ia, Ib, etc.). Each should have its own number. Number the tables in the order in which they are mentioned in the text (Table 1., Table 2, etc).Supply a concise legend that describes the content of the table. Create descriptive column and row headings. Within columns, align data such that decimal points may be traced in a straight line. Use decimal points (periods), not commas, to mark places past the integer (eg, 3.5 rather than 3,5). In a line beneath the table, define any abbreviations used in the table.If a table (or any data within it) was published previously, give full credit to the original source in a footnote to the table. If necessary, obtain permission to reprint from

the author/publisher. The tables should be submitted in Microsoft Word. If a table has been prepared in Excel, it should be imported into the manuscript.

\section{References}

\section{Citation in text}

Please ensure that every reference cited in the text is also present in the reference list (and vice versa). Any references cited in the abstract must be given in full. Unpublished results and personal communications are not permitted in the reference list, but may be mentioned in the text. Citation of a reference as 'in press' implies that the item has been accepted for publication.

\section{Reference links}

Increased discoverability of research and high quality peer review are ensured by online links to the sources cited. In order to allow us to create links to abstracting and indexing services, such as Scopus, CrossRef and PubMed, please ensure that data provided in the references are correct. Please note that incorrect surnames, journal/book titles, publication year and pagination may prevent link creation. When copying references, please be careful as they may already contain errors. Use of the DOI is encouraged.

A DOI can be used to cite and link to electronic articles where an article is in-press and full citation details are not yet known, but the article is available online. A DOI is guaranteed never to change, so you can use it as a permanent link to any electronic article. An example of a citation using DOI for an article not yet in an issue is: VanDecar J.C., Russo R.M., James D.E., Ambeh W.B., Franke M. (2003). Aseismic continuation of the Lesser Antilles slab beneath northeastern Venezuela. Journal of Geophysical Research, http://dx.doi.org/10.1029/2001JB000884i. Please note the format of such citations should be in the same style as all other references in the paper. 
Acceptable references and their placement

Most, if not all, references should first be cited in the Introduction and/or Material and Methods section. Only those references that have been previously cited or that relate directly to the outcomes of the present study may be cited in the Discussion.

Only peer-reviewed, published material may be cited as a reference. Manuscripts in preparation, manuscripts submitted for consideration, and unpublished theses are not acceptable references.

Abstracts are considered unpublished observations and are not allowed as references unless followup studies were completed and published in peer-reviewed journals.

References to foreign language publications should be kept to a minimum (no more than 3 ). They are permitted only when the original article has been translated into English. The translated title should be cited and the original language noted in brackets at the end of the citation.

Textbook references should be kept to a minimum, as textbooks often reflect the opinions of their authors and/or editors. The most recent editions of textbooks should be used. Evidence-based journal citations are preferred.

Reference formatting

References must be identified in the body of the article with superscript Arabic numerals. At the end of a sentence, the reference number falls after the period.

The complete reference list, double-spaced and in numerical order, should follow the Conclusions section but start on a separate page. Only references cited in the text should appear in the reference list.

Reference formatting should conform to Vancouver style as set forth in "Uniform Requirements for Manuscripts Submitted to Biomedical Journals" (Ann Intern Med 1997;126:36-47).

References should be manually numbered.

List up to six authors. If there are seven or more, after the sixth author's name, add et al.

Abbreviate journal names per the Cumulative Index Medicus. A complete list of standard abbreviations is available through the PubMed website: http://www.ncbi.nlm.nih.gov/nimcatalog/journals.

Format for journal articles: Supply the last names and initials of all authors; the title of the article; the journal name; and the year, volume, and page numbers of publication. Do not use italics, bold, or underlining for any part of the reference. Put a period after the initials of the last author, after the article title, and at the end of the reference. Put a semicolon after the year of publication and a colon after the volume. Issue numbers are not used in Vancouver style.

Ex: Jones ER, Smith IM, Doe JQ. Uses of acrylic resin. J Prosthet Dent 1985;53:120-9.

Book References: The most current edition must be cited. Supply the names and initials of all authors/ editors, the title of the book, the city of publication, the publisher, the year of publication, and the inclusive page numbers consulted. Do not use italics, bold, or underlining for any part of the reference.

Ex: Zarb GA, Carlsson GE, Bolender CL. Boucher's prosthodontic treatment for edentulous patients. 11th ed. St. Louis: Mosby; 1997. p. 112-23.

References should not be submitted in Endnote or other reference-generating software. Endnote formatting cannot be edited by the Editorial office or reviewers, and must be suppressed or removed from the manuscript prior to submission. Nor should references be automatically numbered. Please number manually.

See Sample Manuscript. 